ARTICLE

\title{
Three chromosome-scale Papaver genomes reveal punctuated patchwork evolution of the morphinan and noscapine biosynthesis pathway
}

\author{
Xiaofei Yang (10) 1,2,3,9, Shenghan Gao 2,4,9, Li Guo (10) 2,4,9, Bo Wang ${ }^{4}$, Yanyan Jia4 , Jian Zhou6 ${ }^{6}$, Yizhuo Che ${ }^{2,4}$, \\ Peng Jia (1) ${ }^{2,4}$, Jiadong $\operatorname{Lin}^{2,4,7}$, Tun $\mathrm{Xu}^{2,4}$, Jianyong $\operatorname{Sun}^{8} \&$ Kai $\mathrm{Ye} \mathbb{C}^{2,3,4,5,7 凶}$
}

For millions of years, plants evolve plenty of structurally diverse secondary metabolites (SM) to support their sessile lifestyles through continuous biochemical pathway innovation. While new genes commonly drive the evolution of plant SM pathway, how a full biosynthetic pathway evolves remains poorly understood. The evolution of pathway involves recruiting new genes along the reaction cascade forwardly, backwardly, or in a patchwork manner. With three chromosome-scale Papaver genome assemblies, we here reveal whole-genome duplications (WGDs) apparently accelerate chromosomal rearrangements with a nonrandom distribution towards SM optimization. A burst of structural variants involving fusions, translocations and duplications within 7.7 million years have assembled nine genes into the benzylisoquinoline alkaloids gene cluster, following a punctuated patchwork model. Biosynthetic gene copies and their total expression matter to morphinan production. Our results demonstrate how new genes have been recruited from a WGD-induced repertoire of unregulated enzymes with promiscuous reactivities to innovate efficient metabolic pathways with spatiotemporal constraint.

\footnotetext{
${ }^{1}$ School of Computer Science and Technology, Faculty of Electronic and Information Engineering, Xi'an Jiaotong University, Xi'an, Shaanxi, China. ${ }^{2}$ MOE Key Lab for Intelligent Networks \& Networks Security, Faculty of Electronic and Information Engineering, Xi'an Jiaotong University, Xi'an, Shaanxi, China.

${ }^{3}$ Genome Institute, The First Affiliated Hospital of Xi'an Jiaotong University, Xi'an, Shaanxi, China. ${ }^{4}$ School of Automation Science and Engineering, Faculty of Electronic and Information Engineering, Xi'an Jiaotong University, Xi'an, Shaanxi, China. ${ }^{5}$ School of Life Science and Technology, Xi'an Jiaotong University, Xi'an, Shaanxi, China. ${ }^{6}$ College of Life Sciences, Henan Normal University, Xinxiang, Henan, China. ${ }^{7}$ Faculty of Science, Leiden University, Leiden, The Netherlands. ${ }^{8}$ School of Mathematics and Statistics, Xi'an Jiaotong University, Xi'an, Shaanxi, China. ${ }^{9}$ These authors contributed equally: Xiaofei Yang,

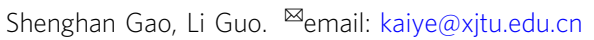


$\mathrm{M}$ etabolic gene cluster (MGC) is a special genetic architecture well known in microorganisms involved in both primary and secondary metabolisms. While MGCs are relatively uncommon in plants, recent studies have discovered over 30 plant secondary metabolites whose biosynthetic pathways are encoded by MGCs, such as thalianol ${ }^{1}$, noscapine ${ }^{2}$, $a-$ tomatine $^{3}$, momilactones ${ }^{4}$, DIMBOA (2,4-dihydroxy-7-methoxy-1, 4-benzoxazin-3-one $)^{5}$ and taxadiene ${ }^{6}$ etc. Furthermore, genomic mining using algorithms such as plantiSMASH ${ }^{7}$ and PhytoClust $^{8}$ have identified many putative plant MGCs ${ }^{9}$. As more plant genomes being sequenced and analyzed, the list of putative plant MGCs is expected to grow, although how many of these MGCs encode functional metabolic pathways awaits investigation. Functionally related genes in plant genomes are typically unlinked. However, MGCs are exceptional raising questions why they are necessary and widely distributed across multiple kingdoms. One hypothesis for cluster formation is co-inheritance arguing that colocalization of genes prevents losing key pathway genes due to recombination events ${ }^{10}$. Another hypothesis is coexpression suggesting that colocalized genes share common promoters and cis-regulatory elements, ensuring a spatially and temporally coordinated expression of biosynthetic enzymes and thus maximizing the yield of end products ${ }^{11}$.

Despite discovering plant MGCs, our knowledge is limited regarding the mechanisms and history of their formation, maintenance, and diversification. Formation of microbial MGCs is often through relocation or duplication of native genes ${ }^{12,13}$, or horizontal gene transfers ${ }^{14,15}$. By contrast, plants are limited to vertical gene transmission, and thus must have their own as-yetunknown mechanisms of gene cluster formation. Understanding the evolutionary history of plant MGCs can explain why many secondary metabolites are exclusive to plants of specific lineage or species. For example, opium poppy remains the sole natural source for opiates used to extract morphine for painkilling drugs $^{16}$, while the anti-cancer drug paclitaxel (trade name Taxol) mainly exists in the bark of Taxus trees ${ }^{17}$. Additionally, some secondary metabolites have apparently undergone convergent evolution in distantly-related plant lineages ${ }^{18}$. Driven by genetic variations, evolution occurs upon selection on traits that benefit organisms under special environment. Therefore, genetic variations and natural selection expectedly play critical roles in the independent evolution of plant MGCs, giving plants a plethora of structurally diverse secondary metabolites of specific bioactivities to support their sessile lifestyle.

Although ample instances of new genes driving the evolution of biosynthetic pathway have been well documented ${ }^{19-21}$, how a full cascaded metabolic pathway evolved among different lineages is largely unknown. Recent studies suggest that plant gene clusters may have evolved through the recruitment of genes from elsewhere in the genome via duplications and neofunctionalization ${ }^{22,23}$, genomic rearrangemen $\mathrm{t}^{24,25}$, transposons ${ }^{26}$, and other unknown mechanisms. Three main evolution models of pathway have been proposed during the last decades, including the forward, backward, and patchwork model. The forward model depicts the evolution of the pathway by recruiting enzymes catalyzing forwardly from earlier steps to later steps ${ }^{27,28}$, while the backward model describes the pathway evolved by acquiring enzymes in a backward fashion, e.g., from later steps to earlier steps ${ }^{29}$. The patchwork model suggests metabolic pathways are assembled by duplicating genes encoding enzymes reacting with diverse substrates ${ }^{30,31}$. An evolutionary model for MGC-encoded plant metabolic pathway is overall lacking due to quite limited comparative analysis of multiple chromosomescale plant genomes that are notoriously difficult to assemble. Without taking genomic synteny and large-scale rearrangement events into account, evolutionary analysis of plant gene cluster is likely prone to misleading conclusions on the evolution of pathway.
Opium poppy (Papaver somniferum L.) is a medicinal plant producing various benzylisoquinoline alkaloids (BIAs) such as morphine and noscapine ${ }^{32}$. BIA biosynthetic pathway is one of the most completely elucidated in plants, with key enzymes identified and functionally validated ${ }^{32-34}$. We and others previously reported that the biosynthetic pathways of morphinan and noscapine were primarily controlled by a BIA gene cluster of $584 \mathrm{~kb}$ in $P$. somniferum genome $\mathrm{e}^{24,25}$, representing one of the largest gene clusters encoding secondary metabolic pathways in eukaryotes. The BIA gene cluster includes a 10-gene cluster for noscapine biosynthesis ${ }^{2}$ and five genes (STORR ${ }^{35-37}$, SALSYN, SALAT, SALR, and THS) for morphinan biosynthesis, and also several genes of unknown functions. Genes encoding the biosynthesis of precursor (S)-reticuline (TYDC, $4 O M T, 6 O M T, B B E$ etc.) as well as converting thebaine to morphine (CODM, $C O R$, and $T 6 O D M)$ are unclustered and dispersed in the genome. The morphinan biosynthetic pathway encoded by a single MGC yet producing two distinct subclasses of BIAs, morphinan and noscapine ${ }^{24,32,35,38-40}$, presents an ideal model to study the complex evolutionary history of plant MGCs in early-diverging eudicots, given that the BIAs have experienced both natural selection and artificial selection by a human being.

In this work, we de novo assemble two chromosome-level genomes of $P$. rhoeas (common poppy) and P. setigerum (Troy poppy) and improve the previous draft $P$. somniferum $\mathrm{HN} 1$ genome ${ }^{24}$ to gain insights into the evolution of BIA gene cluster among Papaver species. The three Papaver species are diverse in terms of morphinan and noscapine production level, and WGD events, therefore providing fresh insights into the evolution of the BIA biosynthetic pathway. Through comparative genomic analysis, we infer the evolutionary history of the morphinan biosynthetic pathway and BIA gene cluster which is most likely explained by the punctuated patchwork model.

\section{Results}

Quantification of noscapine and morphinans in three Papaver species. We evaluated BIA productions of three Papaver species by quantifying the noscapine and morphinans (thebaine, codeine, and morphine) in capsules using the HPLC-MS method (Supplementary Methods 1 and 2). P. somniferum HN1 cultivar $(2 n=22)^{24}$ (Supplementary Fig. 1) accumulated the highest amount of morphinan and noscapine (Supplementary Fig. 2). P. setigerum DCW1 $(2 n=44)$ (Fig. 1a and Supplementary Fig. 1$)^{41,42}$ produced no noscapine, and its production of thebaine, codeine, and morphine were only $6.6 \%, 50 \%$, and $5.5 \%$ of what was produced by $P$. somniferum HN1, respectively (Supplementary Fig. 2). P. rhoeas YMR1 cultivar $(2 n=14)^{43,44}$ (Supplementary Fig. 1) produced a trace amount of morphinan and noscapine (Supplementary Fig. 2 and Supplementary Method 3).

Genome assembly and annotation. To assemble P. setigerum and $P$. rhoeas genomes, we used a combination of sequencing technologies including Oxford Nanopore (ONT) long reads, ONT ultra-long reads, Illumina paired-end reads, and high-throughput chromosome conformation capture (Hi-C) sequencing reads (Supplementary Data 1 and Supplementary Methods 4, 5). The genome assemblies of $P$. setigerum and $P$. rhoeas were highly contiguous with $97.6 \%$ and $87.9 \%$ of genome contigs anchored to chromosomes by using Hi-C scaffolding, respectively (genome assembly size of 4.6 and $2.5 \mathrm{~Gb}$, scaffold N50 values of 211.2 and $329.4 \mathrm{Mb}$, contig N50 values of 65.6 and $5.3 \mathrm{Mb}$ ) (Table 1 and Supplementary Figs. 3-6, Supplementary Data 2, Supplementary Method 6). Facilitated by Hi-C scaffolding, $92.4 \%$ of original $P$. somniferum sequences ${ }^{24}$ were anchored on 11 chromosomes with a scaffold N50 of $249.6 \mathrm{Mb}$, much-improved assembly contiguity 


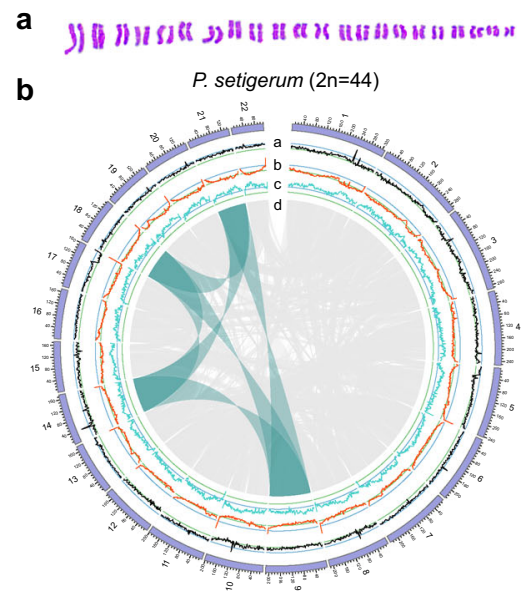

C P. somniferum (One WGD)

P. setigerum (Two WGDs)
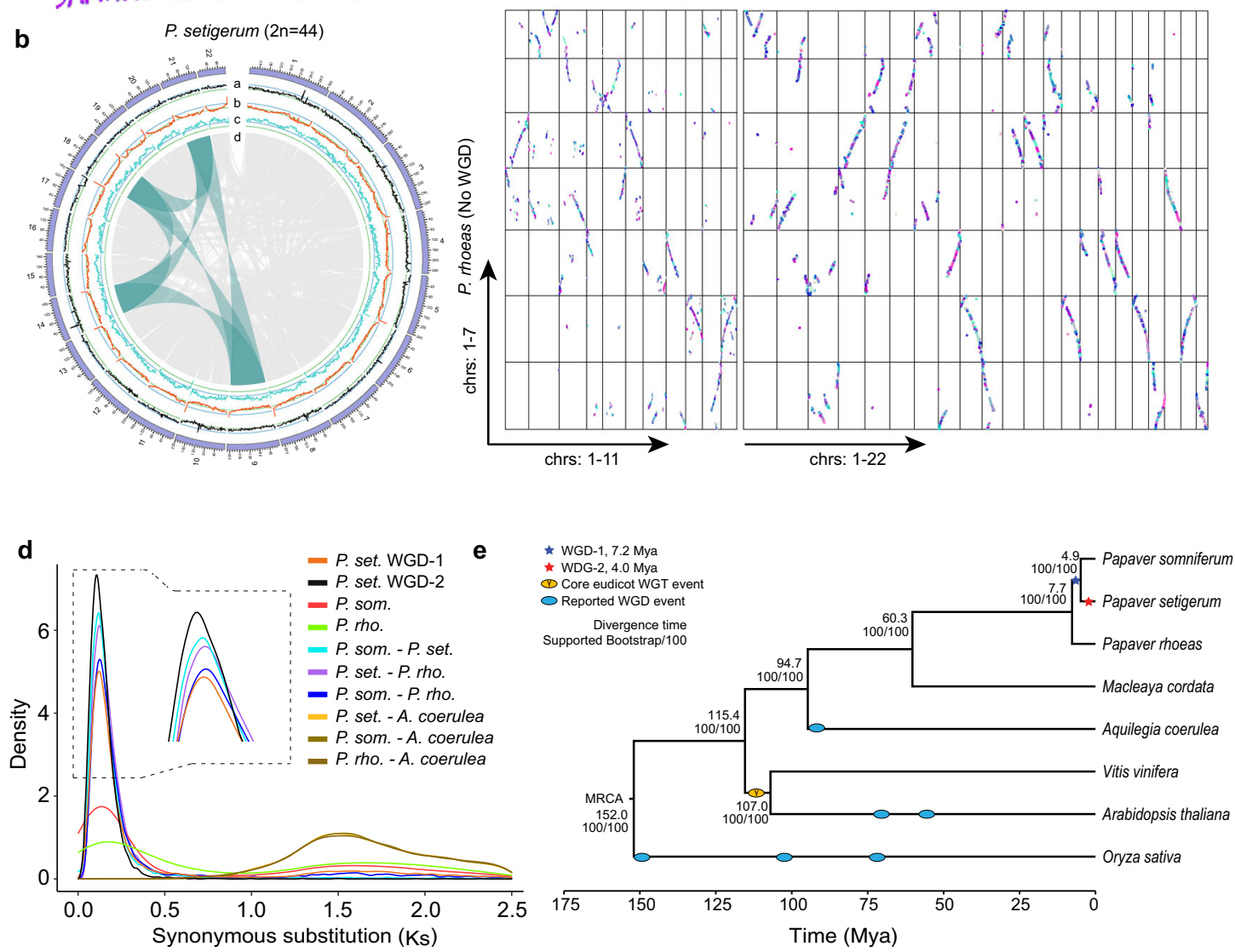

Fig. 1 Genome features of three Papaver species. a Karyotyping shows $P$. setigerum is diploid $(2 n=44)$. b Intraspecies synteny analysis reveals two rounds of whole-genome duplications (WGD) in P. setigerum genome. The tracks $a, b$, and $c$ of the circos plot represent the distribution of gene density, repeat density, and GC density, respectively (calculated in $2 \mathrm{Mb}$ windows). Track $d$ shows genome-wide syntenic blocks detected by $\mathrm{MCScanX} \mathrm{B}^{87}$ where an example of duplications involving four chromosomes as a result of two WGDs was highlighted. Bandwidth is proportional to syntenic block size. $\mathbf{c}$ Dotplots of interspecies syntenic blocks reveal the 1:2:4 syntenic block ratio of $P$. rhoeas, $P$. somniferum, and $P$. setigerum. Each dot indicates a syntenic gene pair detected by MCScanX. Synteny blocks were colored by MCScanX. d Synonymous substitution rate (Ks) density distributions of syntenic paralogs and orthologs, with colored lines representing comparisons among four plant species: $P$. set. ( $P$. setigerum), $P$. rho. ( $P$. rhoeas), $P$. som. ( $P$. somniferum) and $A$. coerulea (Aquilegia coerulea) ${ }^{89}$. For $P$. setigerum, only the reciprocal best matches among the syntenic gene pairs were considered as the pairs from WGD-2 while other syntenic gene pairs were grouped as the pairs from WGD-1. e Inferred maximum likelihood phylogenetic tree by RAxML ${ }^{94}$. Divergence timings and the supported bootstrap values were labeled on the tree. Estimated WGD-1 and WGD-2 timings and the reported whole-genome triplication (WGT)/ WGD timings were superimposed on the phylogenetic tree. MRCA most recent common ancestor, Mya million years ago. Source data underlying b-e are provided as a Source Data file.

over the original version ${ }^{24}$ (Table 1 and Supplementary Fig. 7, Supplementary Data 2, Supplementary Method 6). After reassignment of chromosome IDs, we found the BIA gene cluster was located at chr4 rather than chr11. Benchmarking Universal Single-Copy Orthologs (BUSCO) ${ }^{45}$ evaluation revealed the high genome completeness (92.8-95.3\%) (Table 1, Supplementary Fig. 8). We annotated 41,407 to 106,517 protein-coding genes and 12,429-23,109 non-coding RNAs for the three Papaver species (Table 1 and Supplementary Figs. 9, 10, Supplementary Method 7). Repetitive elements make up more than $70 \%$ for each Papaver genome, and over $50 \%$ of them are long terminal repeat retrotransposons (LTRs) (Table 1 and Supplementary Fig. 11, Supplementary Method 7).

Whole-genome duplication events in three Papaver species. Synteny analysis revealed two rounds of WGDs in P. setigerum but no WGD in $P$. rhoeas, and confirmed the reported single WGD in $P$. somniferum ${ }^{24}$ (Fig. 1 b, $c$ and Supplementary Figs. 12-16, Supplementary Method 8). In addition, a collinearity analysis of three Papaver species with grape (Vitis vinifera $)^{46}$ and ancestral eudicot karyotype genome ${ }^{47}$ (Supplementary Figs. 17 and 18) confirmed a lack of the whole-genome triplication $(\gamma$ event) occurred in core eudicots in Papaver genus.

We next investigated whether $P$. somniferum and $P$. setigerum shared any WGD. Using the estimated divergence time of around 7.7 million years ago (Mya), which is consistent with TimeTree ${ }^{48}$, and mean synonymous substitutions per synonymous site (Ks) of syntenic gene pairs between $P$. somniferum and $P$. rhoeas, we estimated the mutation rate as $8.1 \times 10^{-9}$ synonymous substitutions per site per year for Papaver. Based on this rate and Ks distribution, the $P$. somniferum WGD occurred at around 7.2 Mya, within the range of previous estimation ${ }^{24}$ (Fig. 1d and Supplementary Data 3, Supplementary Method 8). Phylogenomic analysis of eight angiosperms using 48 single-copy orthologs identified by OrthoFinder ${ }^{49}$ indicated $P$. setigerum and $P$. somniferum diverged at around 4.9 Mya (Fig. 1e and Supplementary Method 8), following the divergence of their common ancestor from $P$. rhoeas at around 7.7 Mya. Since the $P$. somniferum WGD predated the divergence of $P$. somniferum and 


\begin{tabular}{|c|c|c|c|}
\hline & P. setigerum & P. rhoeas & P. somniferum \\
\hline \multicolumn{4}{|l|}{ Contig } \\
\hline Total number of contigs & 553 & 3,273 & 61,801 \\
\hline Assembly size (MB) & 4590.03 & 2541.84 & 2709.32 \\
\hline Contig N50 (MB) & 65.57 & 5.29 & 1.74 \\
\hline Contig N90 (MB) & 13.37 & 0.61 & 0.12 \\
\hline Largest contig (MB) & 178.78 & 39.02 & 13.77 \\
\hline \multicolumn{4}{|l|}{ Scaffold } \\
\hline Total number of scaffolds & 381 & 237 & 55,380 \\
\hline Assembly size (MB) & 4590.12 & 2542.27 & 2712.53 \\
\hline Scaffold N50 (MB) & 211.16 & 329.41 & 249.6 \\
\hline Scaffold N90 (MB) & 155.08 & 33.61 & 172.47 \\
\hline Largest scaffold (MB) & 329.11 & 361.76 & 328.07 \\
\hline BUSCO for genome & $94.50 \%$ & $92.80 \%$ & $95.30 \%$ \\
\hline GC content & $36.88 \%$ & $37.94 \%$ & $37.28 \%$ \\
\hline Number of gaps & 172 & 3,036 & 6,421 \\
\hline \multicolumn{4}{|l|}{ Annotation } \\
\hline $\begin{array}{l}\text { Number of protein- } \\
\text { coding genes }\end{array}$ & 106,517 & 41,470 & 55,316 \\
\hline $\begin{array}{l}\text { Supported by RNA-seq or } \\
\text { homologs }\end{array}$ & $100 \%$ & $100 \%$ & $100 \%$ \\
\hline $\begin{array}{l}\text { Supported by } \\
\text { protein family }\end{array}$ & $70.56 \%$ & $70.14 \%$ & $70.05 \%$ \\
\hline Repeat density & $71.55 \%$ & $73.92 \%$ & $76.68 \%$ \\
\hline Number of ncRNA & 23,109 & 12,429 & 12,636 \\
\hline
\end{tabular}

P. setigerum (4.9 Mya), this WGD is essentially the same event as the first WGD (WGD-1) in P. setigerum which has further underwent a lineage-specific WGD (WGD-2) dated around 4.0 Mya (Fig. 1d, e and Supplementary Data 3).

Ancestral genomes and accelerated non-random post-WGD rearrangements. Rearrangements following WGD events are common in plant genome evolution. We are curious about what genome structural changes led to the present-day karyotypes of three Papaver species. We adopted the computational strategy proposed by Sankoff et al. ${ }^{50,51}$ and developed a bottom-up workflow to reconstruct pre- and post-WGD-1 ancestors for three Papaver species (Supplementary Figs. 19, 20 and Supplementary Method 9). Ancestral genome reconstruction relies on accurate genome assembly, and potential misassembly confounds the reconstruction and downstream analysis. Based on the highquality genome assemblies of three Papaver species, the reconstructed pre- and post-WGD-1 ancestral genome had six and eleven protochromosomes, respectively (Fig. 2a and Supplementary Fig. 21). Compared to pre-WGD-1 ancestral genome, $P$. rhoeas likely needed at least five chromosomal fissions and four chromosomal fusions to reach its current structure of seven chromosomes (Fig. 2a and Supplementary Fig. 21). By contrast, $P$. setigerum experienced a much more complex evolutionary history involving two rounds of WGDs and post-WGD rearrangements that finally shaped its karyotype of 22 chromosomes. Shared with $P$. somniferum, $P$. setigerum likely underwent at least 11 chromosomal fissions and 12 chromosomal fusions after WGD-1, and then at least 20 chromosomal fissions and 20 chromosomal fusions following a lineage-specific WGD-2 (Fig. 2a).

We observed a non-random distribution of chromosomal fissions and chromosomal fusions associated with the transformation of the reconstructed pre-WGD-1 protochromosomes to modern chromosomes (Fig. 2). Three chromosomes (chr7, chr16, and chr19) in $P$. setigerum were significantly enriched with fusion events, while chr21 was significantly depleted of them ( $p$-value $<$ 0.05 , z-test, Fig. 2a). Similarly, in pre-WGD-1 ancestor, protochromosome 4 was significantly enriched with fission events, whereas protochromosome 2 was significantly depleted of them ( $p$-value $<0.05, z$-test, Fig. $2 b)$. In addition, the numbers of WGDs and fission events needed to shape the current karyotypes in three Papaver species have a superlinear correlation (Fig. 2b), indicating that post-WGD genome rearrangements might have been accelerated. WGD plays a significant role in the plant secondary metabolism evolution ${ }^{52}$ inspiring us to investigate the function of post-WGD diploidization in Papaver species. The genes around the shuffling breakpoints in $P$. somniferum were enriched in KEGG (Kyoto Encyclopedia of Genes and Genomes) pathways related to isoquinoline and indole alkaloid biosynthesis, as well as the metabolism of amino acids, such as tyrosine and phenylalanine, whereas breakpoint-vicinity genes in $P$. setigerum were enriched with plant-pathogen interactions and environmental adaptation (Supplementary Fig. 22). These results suggest a post-WGD diploidization might have played a part in the optimization of the biosynthetic pathways of alkaloids such as morphinans in the ancestor of $P$. somniferum and $P$. setigerum.

Recruitment of new genes to BIA gene cluster locus. Although the chromosomal shuffling events affected isoquinoline alkaloid metabolism, we did not observe their impact on the BIA gene cluster (Fig. 2a and Supplementary Data 4, 5, Supplementary Method 10). Therefore, we compared three Papaver genomes to identify additional structural variation events potentially leading to the formation of the BIA gene cluster. STORR encodes a fusion protein of a cytochrome P450 and an oxidoreductase (Supplementary Fig. 23), and enables the gateway reaction towards morphinan biosynthesis ${ }^{35-37}$. If the deletion of the intergenic region between pre-fusion modules is the sole event causing STORR fusion, one should expect collinearity between loci encompassing STORR and its pre-fusion modules. However, such collinearity was observed in neither $P$. somniferum nor $P$. setigerum (Supplementary Figs. 12 and 24), intriguing us to hypothesize that STORR evolution may involve additional translocation besides the fusion event with unknown order, the so-called "fusion, translocation" (FT) event.

We then systematically examined two genomic loci implicated in the translocation event of the three species and traced the evolutionary history of STORR. We defined the donor loci as genomic regions syntenic with the $P$. somniferum regions containing the pre-fusion modules, and the recipient loci as genomic regions collinear with the $P$. somniferum regions harboring the STORR locus (Fig. 3a and Supplementary Figs. 25, 26). In addition, donor loci carrying the pre-fusion modules represent the prior state of the FT event, while other donor loci denote the post-state. As for recipient loci, the ones carrying STORR represent the post-state, while the others represent the prior state (Fig. 3a and Supplementary Figs. 25, 26). We found all four types of loci appeared exactly once in $P$. somniferum, but twice in $P$. setigerum, while only the prior states of donor and recipient loci were observed once in $P$. rhoeas (Fig. 3a and Supplementary Figs. 25, 26, Supplementary Methods 11), supporting our hypothesis.

Considering the phylogeny and WGD history among the three species, we proposed a parsimonious evolutionary model to illustrate a burst of genomic rearrangements giving the birth of STORR to the current BIA cluster (Fig. 3b). The ancestor of three species contains the pre-fusion modules at donor loci and an empty recipient locus, which is preserved in $P$. rhoeas. After divergence from $P$. rhoeas, WGD-1 at around 7.2 Mya resulted in two copies of pre-fusion modules, of which one copy was converted to STORR by a FT event and inherited by $P$. somniferum. After divergence from $P$. somniferum, $P$. setigerum 


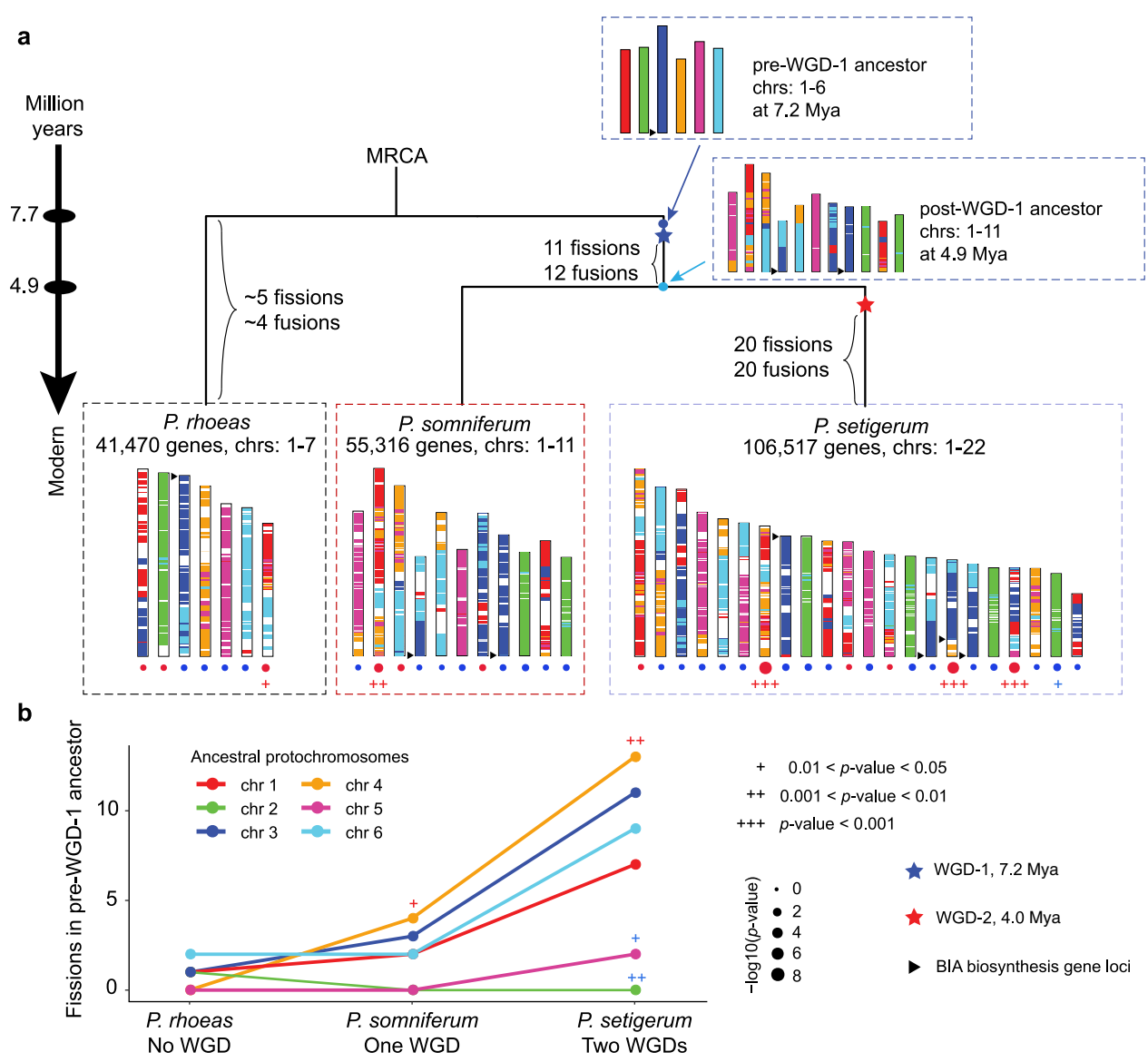

Fig. 2 Ancestral genomes and accelerated non-random post-WGD rearrangements. a Both ancestral and modern genomes were illustrated with a sixcolor code corresponding to protochromosomes of pre-WGD-1 ancestor. Under each modern chromosome, red circles indicate enriched fusions, while blue circles indicate depletion of fusions. MRCA most recent common ancestor, WGD whole-genome duplication, Mya million years ago. $\mathbf{b}$ Number of fissions on each pre-WGD-1 ancestor protochromosomes to shape three modern genomes. The $p$-value of enrichment and depletion were marked as red and blue "+", respectively, $p$-value is calculated by $z$-test. The $p$-values for protochromosome 4 are 0.034 and 0.005 in $P$. somniferum and in $P$. setigerum, respectively; the $p$-value for protochromosome 2 is 0.004 in $P$. setigerum; the $p$-value for protochromosome 5 is 0.02 in $P$. setigerum. Source data are provided as a Source Data file.

underwent a lineage-specific WGD-2 at around 4.0 Mya, yielding two copies of STORR at recipient loci and two copies of prefusion module at donor loci (Fig. 3b). Based on comparative analysis of three Papaver genomes, current computational strategies, and parsimonious assumption, our STORR evolutionary model represents a working hypothesis. The inclusion of sequencing data from additional species may lead to an updated evolutionary model or alternative hypotheses.

We next investigated the evolutionary history of the remaining genes in the BIA gene cluster. Unlike previous analyses focusing on gene trees ${ }^{53}$, we integrated evidence of multiple sources from synteny, phylogeny, protein sequence alignments, and WGD (Fig. 4a and Supplementary Figs. 27-36, Supplementary Methods 11,12) and found that four genes (PSSDR1, CYP82X1, CYP719A21, and PSMT1) in noscapine branch and two (SALSYN and $S A L R$ ) in morphinan branch were already present in MRCA of three Papaver species (Fig. 4b). Three genes (STORR, SALAT, and THS) in the morphinan branch were assembled before $P$. somniferum diverged from $P$. setigerum, while the assembly of noscapine branch was completed in P. somniferum via adding six new genes (PSCXE1, CYP82X2, PSAT1, PSMT2, CYP82Y1, and PSMT3) through lineage-specific duplications (Fig. $4 \mathrm{~b}$ and Supplementary Figs. 27-35, Supplementary Method 11). Specifically, we inferred five genes including PSCXE1, PSAT1, PSMT3, $S A L A T$, and THS may be assembled into the BIA gene cluster by putative dispersed duplications (the gene and their inferred original copy are non-syntenic and not adjacent) while CYP82X2 was likely generated from a tandem duplication of CYP82X1 (Fig. 4b and Supplementary Figs. 27-35). Considering the difficulty of distinguishing dispersed duplication from old tandem duplication with gene deletions, we do not rule out the possibility that PSCXE1, PSAT1, PSMT3, SALAT, and THS were assembled into BIA gene cluster by ancient tandem duplications with followup gene deletions (Supplementary Fig. 37).

Clustered BIA genes are co-regulated and evolved in a coordinated manner. Assembly of 15 genes encoding two distinct pathways into a compact gene cluster is striking. The genetic components of the BIA pathway should allow the biosynthesis of morphinan and noscapine albeit at their original loci before clustering, raising questions on the necessity of gene clustering in evolution. In fact, besides the BIA gene cluster, the phenomenon of gene clustering has been reported for multiple plant secondary metabolic pathways ${ }^{54}$. This increasingly common theme suggests that plants, with a sessile lifestyle, are under selection pressure to evolve a special genetic architecture to facilitate their adaptation to environmental stimuli ${ }^{55}$, consistent with our gene family analysis (Supplementary Data 6-8). Recently, we and others have reported that gene clustering likely enables the coordinated 

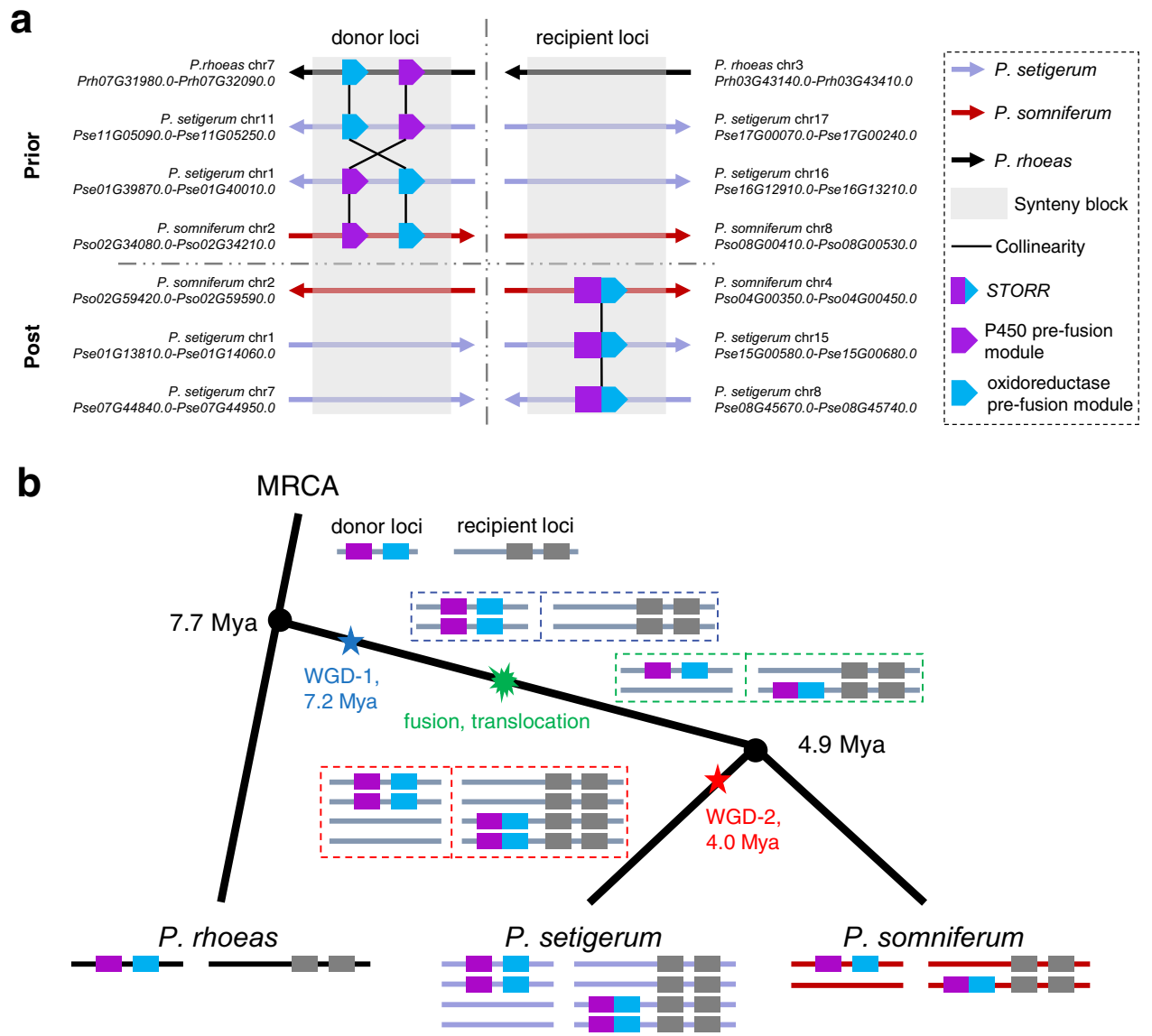

Fig. 3 Evolutionary history of STORR. a The collinearity of genes in the donor loci and the recipient loci showing both prior and post states of FT event in three Papaver species supporting the "fusion, translocation" (FT) event leading to STORR formation at BIA gene cluster. The directions of arrows indicated the chromosome from $5^{\prime}$ to $3^{\prime}$. The dash lines indicate the boundary between donor loci and recipient loci, and the boundary between prior states and post states. The detailed diagram with the genomic context was shown in Supplementary Fig. 25. b A proposed evolutionary model for the birth of STORR at BIA gene cluster raised from a series of whole-genome duplications (WGD), "fusion, translocation" events. Purple and blue colors denote the cytochrome P450 and oxidoreductase modules. MRCA most recent common ancestor, WGD whole-genome duplication, Mya million years ago. Source data underlying a are provided as a Source Data file.

expression of metabolic enzymes for BIA biosynthesis in opium poppy stem and root tissues ${ }^{24,56}$. However, it remains unclear how Papaver species achieve the coordinated BIA gene expression by evolving this gene cluster. To approach this question, we dissected the tissue-specific expression profiles of the BIA gene cluster and its closest paralogs within $P$. somniferum and $P$. setigerum (Supplementary Method 13). For STORR gene-related loci, we found that the donor locus had low expression levels across all tissues among three species while the recipient locus exhibited high expression in the stem where morphinan biosynthesis primarily occurs ${ }^{24}$ (Fig. 5a and Supplementary Fig. 38). Expression pattern of the other eight new genes at the BIA gene cluster and their ancestral copies suggests that except for a putative tandem duplicated gene CYP82X2, all were duplicated from putative ancestral copies at remote origin loci of largely low and non-tissue-specific expression to the BIA gene cluster displaying a high and coordinated expression in stem (Supplementary Fig. 38). The fact that genes at recipient prior locus were also expressed in stems (Fig. 5a and Supplementary Fig. 38) indicated that new genes arrived at the BIA gene cluster were perhaps preequipped with a stem-specific promoter or regulatory elements (Supplementary Data 9 and 10).

We further sought potential epigenetic factors contributing to the co-expression of BIA gene cluster. Hi-C data showed $P$. somniferum noscapine branch and morphinan branch were in two different chromatin blocks physically interacting likely through a chromatin loop as shown by $\mathrm{Hi}-\mathrm{C}$ contact maps (Supplementary Fig. 39 and Supplementary Method 14), indicating simultaneous regulation of both pathway branches via an epigenetic regulatory mechanism. Although P. setigerum lineagespecific WGD-2 created a second copy of the morphinan branch, only one copy is highly expressed in stem. The highly expressed copy on chr15 has significantly more chromatin contacts than the lowly expressed copy on chr8 ( $p$-value $=6.18 \mathrm{e}-4$, Fig. 5b, $\mathrm{c}$ and Supplementary Fig. 40). Although coding regions of five genes involved in morphinan biosynthesis remain intact in both copies in $P$. setigerum, the promoter regions for five genes in the lowly expressed copy were eroded but were overall maintained by the highly expressed copy (Fig. 5d and Supplementary Fig. 41). This is unexpected because the chance of promoter erosion occurring at either copy should be almost equal unless the locus-specific promoter conservation is favorably selected. Thus, such findings indicated that the observed physical clustering of co-expressed and co-regulated genes is under strong positive selection for a beneficial trait, which keeps the cluster evolved in a coordinated manner.

Total expression of all gene copies contributes to morphinan production. Copy number variation of BIA biosynthetic genes 


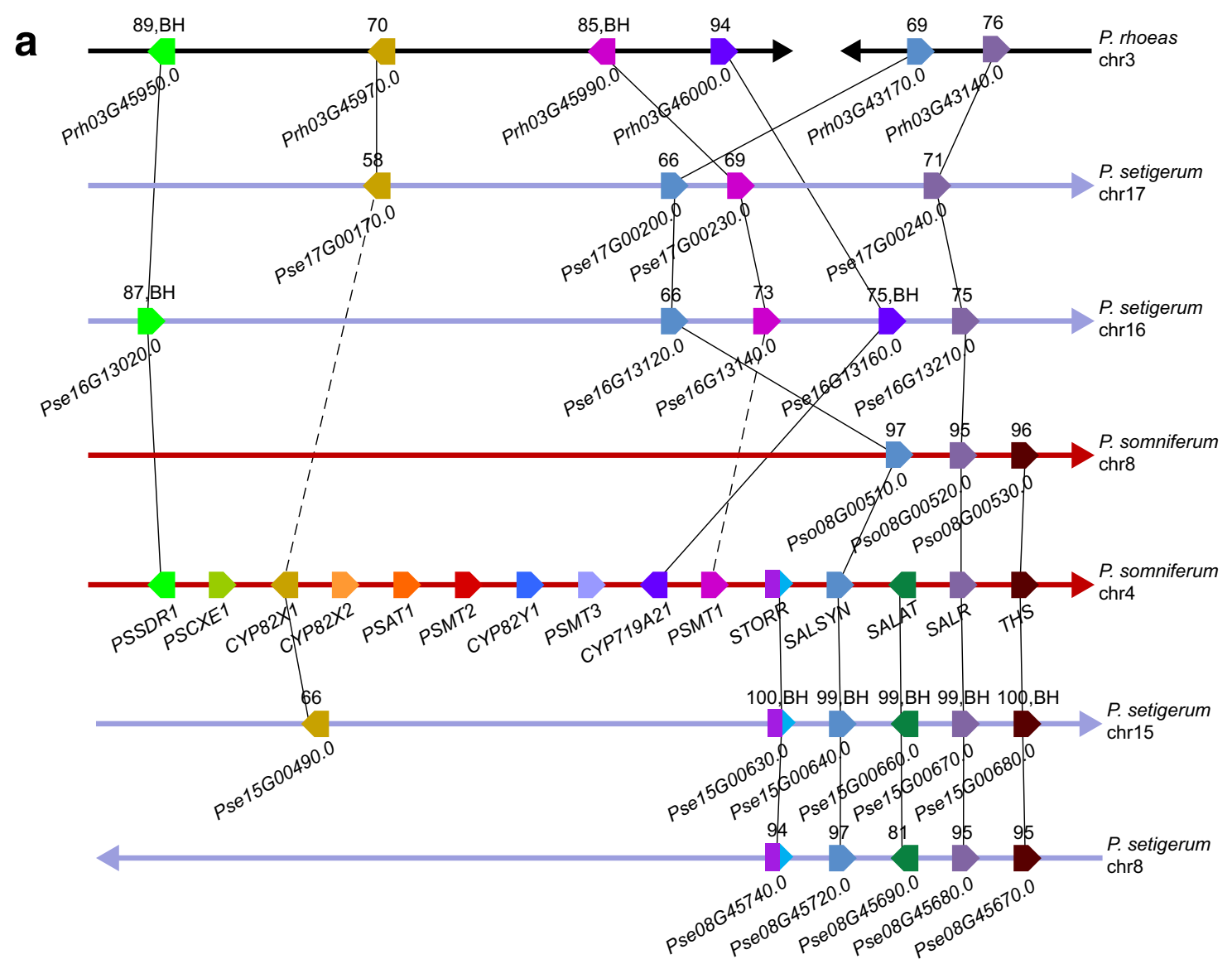

b

BIA gene cluster

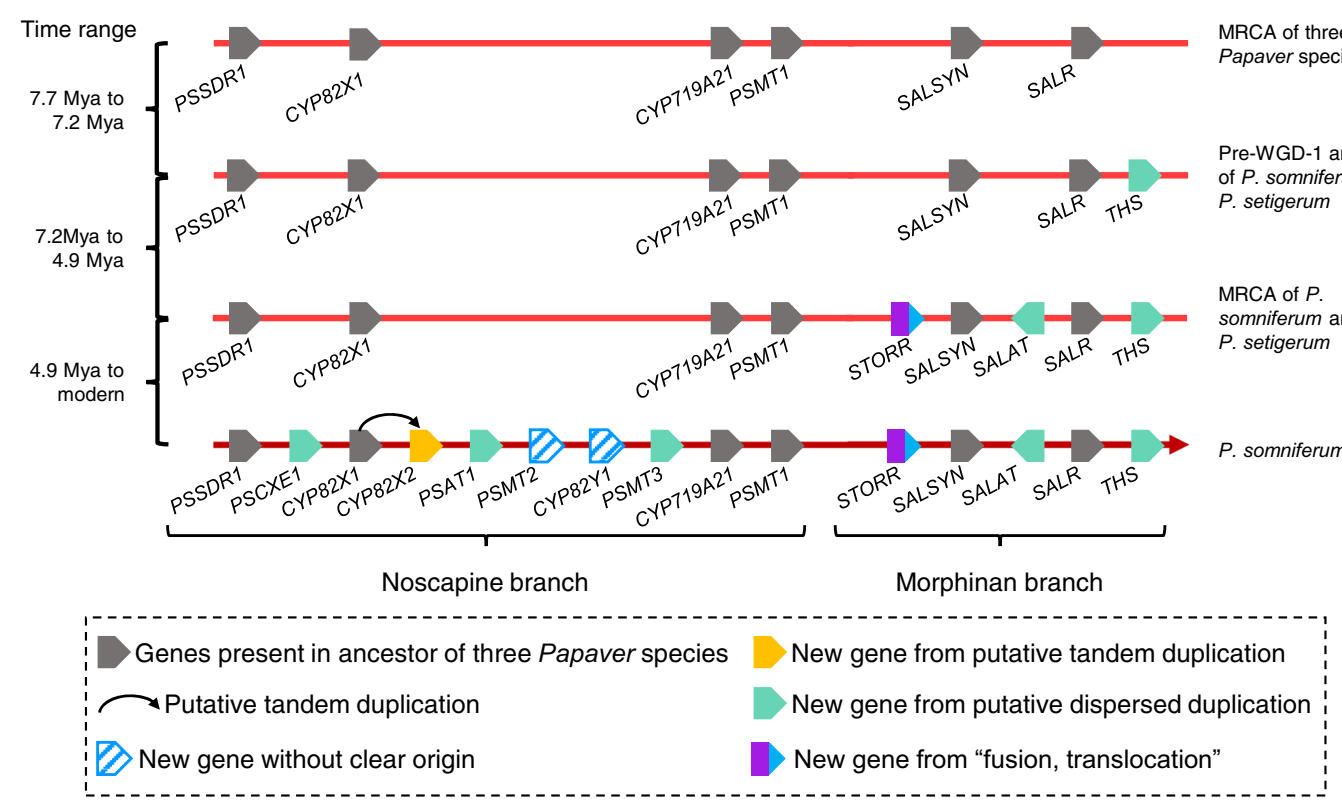

Fig. 4 Evolutionary history of benzylisoquinoline alkaloid (BIA) gene cluster. a The syntenic relations at BIA gene cluster in three Papaver species. Solid lines denote the syntenic relations detected by MCScanX, and the dash lines denote the top BlastP hits. Different colors denote different genes. The gene name or ID is labeled for each gene. The numbers above the genes represent sequence identity levels from BlastP. The directions of arrows indicate the chromosome from $5^{\prime}$ to $3^{\prime}$. BH best hit. b Summary of BIA gene cluster evolution. Putative tandem duplication as a structural variation event producing identical adjacent segments while the other non-locally duplicated ones are "putative dispersed duplication". MRCA most recent common ancestor, WGD whole-genome duplication, Mya million years ago. Source data underlying a are provided as a Source Data file. 
a
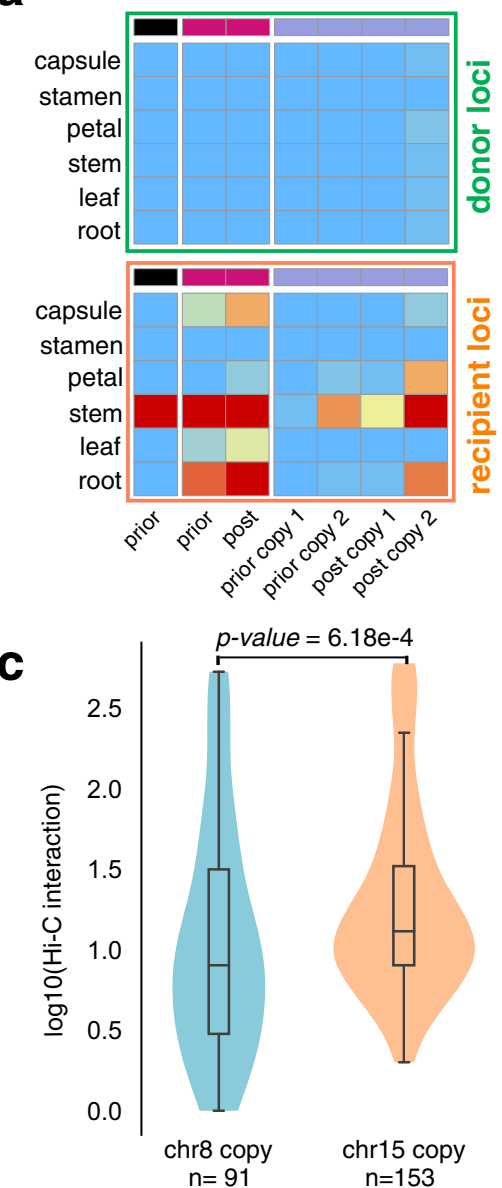

b

Hi-C contact map of morphinan branch copies in $P$. setigerum
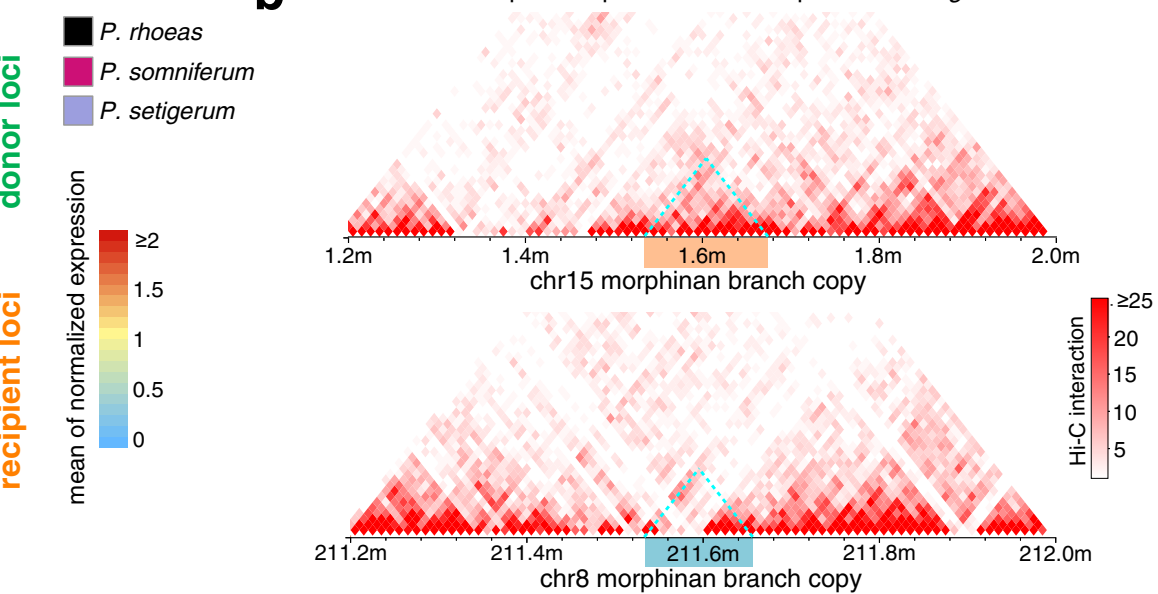

chr8 morphinan branch copy

d

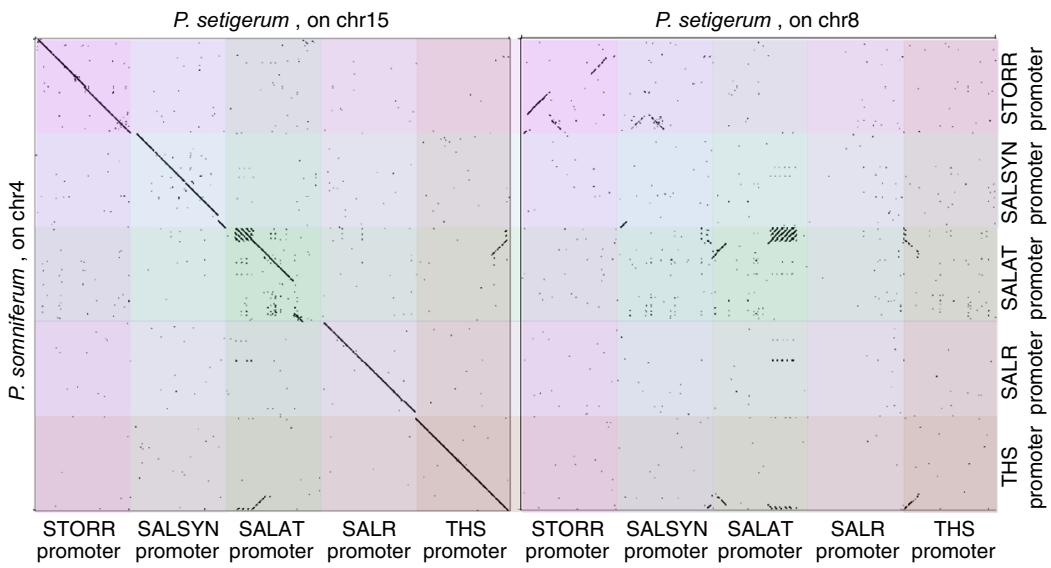

Fig. 5 Clustered BIA genes are co-regulated and evolved in a coordinated manner. a The heatmap of the average normalized expression level of genes at the donor and the recipient loci related with STORR in six tissues of three Papaver species. Details of the gene expressions related to BIA genes were shown in Supplementary Fig. 38. b Hi-C interaction heatmap of regions including two copies of morphinan gene cluster on chr15 (top) and chr8 (down) of $P$. setigerum, shown at a resolution of $10 \mathrm{~kb}$. The morphinan branch gene regions are marked with colored boxes. $\mathbf{c}$ The comparison of the interactions between two morphinan gene copies in $P$. setigerum. The $p$-value is calculated by a two-sided Wilcoxon rank-sum test. For the boxplot, the centerline, median; box limits, upper and lower quartiles; whiskers, data range. $n$ is the number of bins. $\mathbf{d}$ Dotplots of STORR, SALSYN, SALAT, SALR, and THS promoter sequences between $P$. somniferum (one copy) and $P$. setigerum (two copies). Promoter is defined as $2 \mathrm{~kb}$ upstream region of the transcription start site. Source data are provided as a Source Data file.

has been positively correlated with the production of morphinans in $P$. somniferum cultivars in a previous genomic study ${ }^{56}$, indicating a potential gene dosage effect. However, we found the extra copy or copies of STORR, SALSYN, SALAT, and SALR in P. setigerum due to WGD-2 did not lead to more abundant production of morphinans compared to P. somniferum (Fig. 6 and Supplementary Fig. 2). Moreover, the eroded promoters of one copy of the morphinan gene cluster in $P$. setigerum caused low expression, while the intact promoter of the second copy maintained high expression of genes (Supplementary Method 13). Therefore, the copy number is not the only factor affecting BIA productions, and gene expression may also play an important role. We systematically compared the copy number and total expression of all copies of BIA genes encoding enzymes for the morphinan biosynthetic pathway in the three species. We observed that four genes encoding key enzymes, including STORR, SALAT, THS, and T6ODM, were missing in the corresponding syntenic blocks of $P$. rhoeas YMR1 (Fig. 4, Supplementary Fig. 42), probably leading to its negligible amount of three morphinans compared to P. somniferum HN1 (Fig. 6 and Supplementary Fig. 2). By contrast, compared to P. somniferum HN1, $P$. setigerum DCW1 carries twice as many copies of STORR,
SALSYN, SALR, and SALAT, and equal copy number of THS and T6ODM, but fewer copies of COR and CODM catalyzing the final step to codeine and morphine production (Fig. 6 and Supplementary Fig. 42, Supplementary Data 5). The pure difference of gene copy numbers between the two species could not explain the large gap in their morphinan productions. Thus, we compared the total expression of all copies of each morphinan branch gene in the stem where morphinan biosynthesis primarily takes place $^{24,56}$, and found their expression in P. somniferum HN1 was at least twice as high as that in P. setigerum DCW1 (Fig. 6 and Supplementary Fig. 42, Supplementary Data 5). As the morphinan biosynthesis occurs in a cascade fashion, we expect the total expression of all copies of each gene and its copies along the pathway to yield an amplified difference in the gross production of the final products, which is exactly what we observed in the two Papaver relatives (Fig. 6). As such, these results suggest the morphinan production is attributed to the total expression of all copies of key genes besides their copy numbers. Finally, distinct copy numbers for CODM, T6ODM, and COR have been observed among three Papaver species (Fig. 6 and Supplementary Fig. 42) and among cultivars of $P$. somniferum ${ }^{56}$, suggesting that dosage of these genes is possibly under positive selection. 


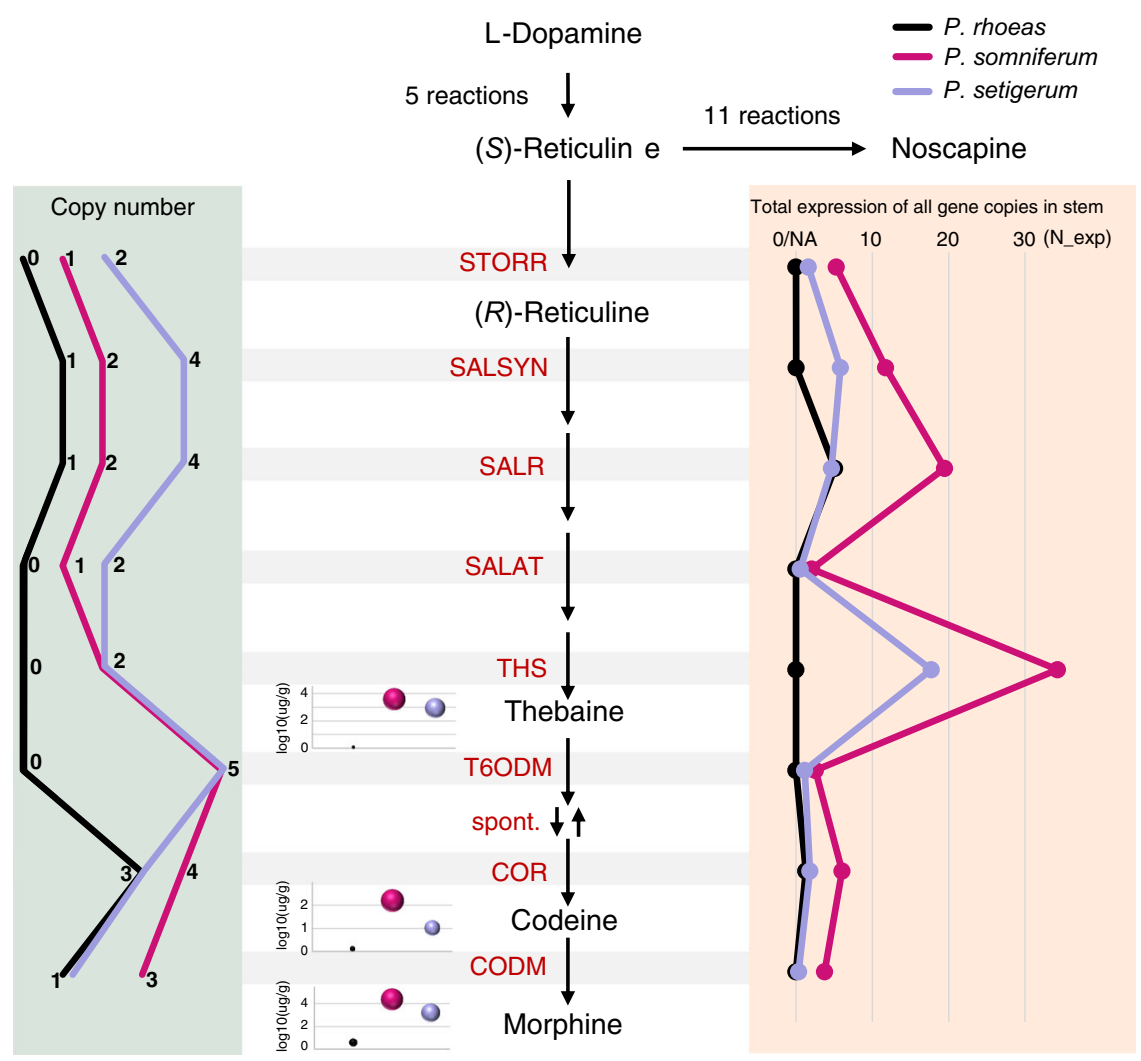

Fig. 6 Copy number and expression of genes on morphinan biosynthetic pathway. Correlation of morphinan production with copy number and expression of morphinan biosynthesis genes in three Papaver species. Copy numbers of morphinan biosynthesis pathway genes were shown in a line plot on left. Production levels of thebaine, codeine, and morphine were shown in bubble plots, and the detail numbers were shown in Supplementary Fig. 2. The total expression of all gene copies in the stem was shown in a line plot on right. N_exp normalized expression. Source data are provided as a Source Data file.

\section{Discussion}

Gene clustering, a frequent genetic paradigm in microbial metabolic pathways, has long been thought as a rarity in higher eukaryotes such as land plants. Until recently, with the burst of plant genome sequencing projects and advancement of bioinformatic tools, biosynthetic pathways for over 30 plant natural products are known to be encoded by gene clusters ${ }^{55}$, including A. thaliana (e.g., thalianol ${ }^{25}$, opium poppy (e.g., morphinan $)^{2,24}$, maize (e.g., DIMBOA $)^{57}$, rice (e.g., momilactones) ${ }^{4}$, and tomato (e.g., $\alpha$-Tomatine $)^{3}$, etc. Our studies on opium poppy, and recent examples shown by other groups ${ }^{56}$ have demonstrated that the formation and diversification of biosynthetic gene clusters in plants have been made possible largely by gene gain and loss through structural variations ${ }^{58}$.

In this study, the contribution of large-scale genome variations, e.g., WGD and subsequent genome rearrangements, to the rise and divergence of biosynthetic pathways is well illustrated by the reconstructed evolutionary history of STORR and other key BIA genes. Three high-quality Papaver genomes and their distinct WGD status enabled us to infer the origins and steps leading to the current BIA gene cluster. We showed that duplication was a major factor contributing to the formation of the BIA gene cluster. The origin and putative type of the duplications were inferred based on synteny, WGD, and protein sequence alignments analysis of three Papaver genomes. Alternative explanations of the BIA gene cluster formation such as "old tandem duplications" are equally possible but shall be tested with more sequencing data and genome analyses. Interspecies comparison of the morphinan production revealed that total expression of gene copies of BIA genes contributed to the morphinan production. Consistently, the importance of gene copies for the BIA biosynthetic genes was also shown by an intraspecies comparison of nine P. somniferum cultivars ${ }^{56}$. Therefore, gene copies are pivotal to both intraspecies and interspecies variation of morphinan production in Papaver.

Papaver species produce diverse types of natural products besides morphinans and noscapine. For example, MenéndezPerdomo et al. summarized 44 authentic BIAs in P. somniferum, including salutaridine, papaverine, and narcotoline $e^{59}$, and Grauso et al. summarized 128 organic compounds isolated from $P$. rhoeas, including leucine and rhoeadine ${ }^{60}$. The fact that biosynthetic pathways of the non-morphinan and non-noscapine compounds are poorly understood in Papaver species limited our further investigation of their metabolic pathways in this study. Thus, we focused on the evolutionary steps leading to the efficient production of the morphinans and noscapine in this interspecies comparison. Expectedly, $P$. rhoeas which produces a trace amount of morphinans and noscapine lacks a bona fide BIA gene cluster. We found that $P$. rhoeas has a primitive metabolic pathway leading to the formation of morphinan precursors such as dopamine, 4-Hydroxyphenylacetaldehyde, and (S)-Norcoclaurine encoded by TYDC (PrhUNG23530.0) and TryAT (Prh05G30560.0), NCS (PrhUNG12800.0), respectively. Given the phylogenetic relationships of the three Papaver spp., it suggests that an ancient repertoire of unregulated enzymes with promiscuous reactivities might be already present in Papaver to allow the production of morphinans at minimal levels. WGD-1 apparently energized the ancestral genome and triggered chromosomal rearrangements for boosting the production levels of beneficial metabolites. As a result, a set of new genes originated from fusions and gene duplications were recruited to a locus preequipped with desired regulatory elements, leading to the 
formation of the BIA gene cluster. The formation of bifunctional STORR enzyme as a result of gene fusion channels metabolic flow preferentially into the morphinan pathway. Recruitment of subsequent enzymes for both morphinan and noscapine pathways as they were branching included neither forwardly nor backwardly evolving progression of evolutionary steps, rejecting both hypotheses of forward pathway evolution ${ }^{61}$ and retrograde evolution of biochemical pathway ${ }^{29}$. Instead, once a relatively large repertoire of enzymes becomes available after WGD-1, duplicated promiscuous enzymes can be readily recruited from various genomic regions into a locus pre-equipped with tissue-specific regulatory elements to maximize flux through morphinan and noscapine pathways, as elegantly explained by the patchwork model, which describes the way of recruitment of enzymes in a pathway like a patchwork manner ${ }^{30,31,62}$. There are extensive instances supporting the patchwork model in bacteria. For example, the pathway for degradation of pentachlorophenol, in Sphingomonas chlorophenolica ${ }^{63}$ and metabolic pathway for 2CNB degradation in Pseudomonas stutzeri ${ }^{64}$. By contrast, an evolutionary model for metabolic pathways in eukaryotes is largely unkonwn ${ }^{54}$. We presented evidence that the evolution of the BIA biosynthetic pathway encoded by a metabolic gene cluster, could be well explained by the patchwork model.

With some exceptions, metabolic gene clustering often enables a coordinated expression of the biosynthetic genes in a tissuespecific manner. We showed in this study that post-WGD genome rearrangement played a major role in achieving this tissuespecific co-expression of the morphinan gene cluster for $P$. somniferum and P. setigerum. We showed that new genes were recruited from lowly expressed genomic loci to the pre-configured locus with a stem-specific expression. It remains a mystery why such a low entropy of tightly packed gene cluster ever exists and evolves coordinative, as it is common to find genes at various genomic loci encoding the same pathway, functionally linked via gene co-regulation. In fact, unclustered biosynthetic genes are common in plants that somehow are co-expressed in specific tissues. One example is the flame lily (Gloriosa superba) colchicine biosynthetic pathway encoded by genes showing no apparent genomic clustering but strongly co-expressed in seeds and corms $^{65}$. For opium poppy, genes such as CODM and T6ODM encoding the tailoring enzymes in morphinan biosynthesis are also unclustered but co-expressed with clustered core genes encoding the morphinan pathway even though located on different chromosomes ${ }^{24,56}$. Among plant metabolic gene clusters, the genetic architecture of a core gene cluster plus several peripheral or satellite genes is rather common 9,55 . The gene clusters can be continuous without any intervening genes such as avenacin (oat) gene cluster ${ }^{34}$, while some have genes of unknown function separating the cluster genes such as thalianol ${ }^{25}$, $\operatorname{DIMBOA}^{57}$, and morphinan gene cluster ${ }^{2,24}$. There are also biosynthetic pathways such as tomatine, morphine, and cucurbitacin $\mathrm{C}$ encoded by a core cluster with satellite genes or cluster ${ }^{55}$. The morphine biosynthetic pathway is composed of a core cluster coupled with several peripheral genes (in trans) or satellite gene groups ${ }^{24}$. Recent studies also show that collinearity exits within a single gene cluster such as the noscapine gene cluster where the cluster is organized in modules that correspond to early, middle and late steps of the pathway ${ }^{2}$. The diverse architecture of metabolic gene clusters shows the evolutionary history of gene clustering for secondary metabolic pathways is complex and species-specific, involving complicated genomic variations and selection processes. Yet how plant biosynthetic genes, either clustered or dispersed in genome, are co-regulated at both epigenetic and transcriptional levels remains largely unknown and needs further investigation. We presented substantial evidence that epigenetic regulations and a non-random erosion of cis-elements within the gene cluster on chr8 in $P$. setigerum underlies the efficient biosynthesis of BIA. The fusion protein STORR couples two reactions, forming the core of a socalled "metabolon" together with co-expressed subsequent morphinan biosynthetic enzymes, to maximize the efficiency of morphinan production but minimize diffusion of toxic intermediate products. The lately formed noscapine pathway joined the locus, perhaps exploiting the optimized regulatory elements and chromatin-level regulation, further expanding the production line of the secondary metabolite factory.

Finally, as we and others have reported the structural variants such as copy number variants within morphinan biosynthetic genes $^{24,56}$, opium poppy genome as well as its morphinan gene cluster are not static and probably still evolving given the natural and human artificial selection process. Thus, it would be interesting to observe how biosynthetic pathways continue to evolve due to human selection, and whether biosynthetic genes that are clustered but did not co-express, and genes that co-expressed but non-clustered will have a converged or diverged evolutionary trajectory in future opium poppy crops.

\section{Methods}

Plant materials, DNA, and RNA isolation. P. setigerum variety DCW1, $P$. rhoeas variety YMR1, and $P$. somniferum variety HN1 were grown in Azalea pots in a growth chamber with $16 \mathrm{~h}$ of light located at Xi'an Jiaotong University Laboratory of BioData Sciences. Genomic DNA was extracted from fresh leaves (the four uppermost ones) harvested from 6-weeks-old seedings of three Papaver species. For Illumina and regular Oxford Nanopore (ONT) sequencing, high molecular weight (HMW) genomic DNA was prepared by the CTAB method and purified with QIAGEN $^{\circledR}$ Genomic kit (Cat\#13343, QIAGEN). For ultra-long ONT sequencing, ultra-high molecular weight DNA was extracted by the SDS method ${ }^{66}$ handled gently to sustain the length of DNA. For transcriptome sequencing (RNA-seq), Total RNA was extracted from the six tissue types on the first day of anthesis: root leaves, stem (the 2 -cm-long part just underneath the capsule), capsule, petal, and stamens, using TRIzol reagent (TIANGEN). RNA integrity was determined using regular agarose gel electrophoresis, Nanodrop (ThermoFisher Scientific), and Agilent 2100 Bioanalyzer (Agilent Technologies). RNA sample of high quality (OD260/280 within range $[1.8,2.2], \mathrm{OD} 260 / 230 \geq 2.0, \mathrm{RIN} \geq 8,>1 \mu \mathrm{g}$ ) was used to construct the sequencing library.

Genome and transcriptome sequencing. DNA and RNA sequencing in this study were performed using sequencers at Nextomics Inc. (Wuhan, China). For ONT sequencing, about 4 and $10 \mu \mathrm{g}$ high molecular weight DNA was used for regular and ultra-long library construction, respectively. About $700 \mathrm{ng}$ (regular) and $800 \mathrm{ng}$ (ultralong) DNA libraries were sequenced on a Nanopore PromethION sequencer. A total of ten and eight ONT cells were used for $P$. setigerum and $P$. rhoeas, respectively. For Illumina paired-end sequencing, a total of $1.5 \mu \mathrm{g}$ DNA was used to construct the library and the libraries were sequenced by using the Illumina NovaSeq platform to generate $150 \mathrm{bp}$ paired-end reads with insert size around $400 \mathrm{bp}$. Hi-C library construction was performed according to a $3 \mathrm{C}$ protocol established for maize ${ }^{67}$. Briefly, $5 \mathrm{~g}$ fresh leaves were fixed with $1 \%$ formaldehyde solution in MS buffer $(10 \mathrm{mM}$ potassium phosphate, $\mathrm{pH} 7.0,50 \mathrm{mM} \mathrm{NaCl}$; $0.1 \mathrm{M}$ sucrose), and homogenized with liquid nitrogen, and then subjected to nuclei isolation and enrichment. Chromatin was digested for $16 \mathrm{~h}$ with $400 \mathrm{U}$ HindIII restriction enzyme (New England Biolabs) at $37^{\circ} \mathrm{C}$. DNA ends were labeled with biotin and DNA ligation was performed by adding T4 DNA ligase (New England Biolabs, USA) and incubation at $16^{\circ} \mathrm{C}$ for 4-6 h, followed by reverse cross-linking using proteinase K (Promega, USA). Purified DNA was fragmented to a size of 300-500 bp, and DNA fragments labeled by biotin were finally separated on Dynabeads ${ }^{\circledR}$ M-280 Streptavidin (Life Technologies, USA). $\mathrm{Hi}-\mathrm{C}$ libraries were controlled for quality and sequenced on an Illumina Novoseq sequencer. For RNA-seq, a total amount of $1 \mu \mathrm{g}$ RNA was used for library construction with the TruSeq RNA Library Preparation Kit (Illumina, USA). The RNAseq libraries were sequenced on an Illumina NovaSeq platform and paired-end reads of $150 \mathrm{bp}$ were generated.

Genome assembly. To obtain chromosome-scale genome assemblies, all sequencing reads were first subjected to quality control. The ONT reads were assembled into primary contigs by using NextDenovo (v2.2) ${ }^{68}$ software, upon which we next performed three rounds of polishing with ONT reads and another round of polishing using Illumina paired-end reads by Nextpolish (v1.2.0 ${ }^{69}$ to yield highquality contigs. Redundant sequences in the polished contigs were removed by purge_dups ${ }^{70}$. Then, breakhic (v1.1) (https://github.com/wtsi-hpag/scaffHiC) was used to identify assembly breakpoints of polished contigs by screening paired $\mathrm{Hi}-\mathrm{C}$ reads. Finally, 3d-DNA (v180922) 71 pipeline was used to reorder and anchor contigs into scaffolds and chromosomes. To assess genome completeness, we 
applied BUSCO (Benchmarking Universal Single-Copy Orthologs) (v3) analysis using the plant early release version (v1.1bl, release May 2015) ${ }^{45}$. In addition, we aligned the Illumina paired-end reads to the final genome assemblies, and detected SNP and Indels by GATK (v4.1.8) 72 to evaluate the base accuracy of the assemblies.

Genome annotation. We used Repbase ${ }^{73}$ and the species-specific de novo repeat library constructed by RepeatModeler (vopen-1.0.8) to annotate the repeat DNA sequences in three Papaver species. RepeatMasker (vopen-4.0.7) was applied to annotated the repeat elements. In addition, we applied LTR_Finder (v1.1 $)^{74}$, LTRharvest (v1.5.9) $)^{75}$, and LTR_retriever (v2.8.5) ${ }^{76}$ to detect LTR elements. Protein-coding genes were predicted using the MAKER2 pipeline (v2.31.8 $)^{77}$ integrating evidence-based and ab initio gene predictors. The evidence includes SwissProt (downloaded in Jan. 2020), protein sequences of A. thaliana ${ }^{78}$, Beta vulgaris ${ }^{79}$, and Vitis vinifera ${ }^{46}$, and transcripts assembled by Trinity (v2.1.1) $)^{80}$. Three ab initio gene predictors include AUGUSTUS (v3.3) ${ }^{81}$, SNAP (v2006-07-28) ${ }^{82}$, and GeneMark_ES (v3.48) ${ }^{83}$. Predicted genes were evaluated in terms of whether they were supported by transcript or protein homolog, the annotation edit distance (AED), and the exon AED (eAED). We kept the predicted genes with AED $<0.5$, eAED $<$ 0.5 , and ones supported by transcript or protein homologs. The function domains of the protein-coding genes were annotated by using interProScan with default parameters ${ }^{84}$. Non-coding RNAs (ncRNAs) were annotated using $\mathrm{cmscan}$ from INFERNAL (v1.1.2) ${ }^{85}$ package based on Rfam database (v14.1) ${ }^{86}$

Whole-genome duplication identification. The syntenic analysis was performed by $\mathrm{MCScanX}^{87}$ with default parameters from top-five BlastP hits. Within each Papaver genome, the proportion of genes with WGD/segmental duplication types and the widespread and well-maintained copy numbers of the syntenic blocks indicate no WGD in $P$. rhoeas, single WGD in P. somniferum, and two WGDs in $P$. setigerum. In addition, comparison among three Papaver genomes, between ancestral eudicot karyotype (AEK) ${ }^{47}$ and each Papaver genome, as well as between Vitis vinifer ${ }^{46}$ and each Papaver genome provides other evidence for confirming the number of WGDs in three Papaver genomes.

Phylogenomic analysis and divergence time estimation. We applied OrthoFinder (v2.3.4) ${ }^{49}$ to detect single-copy orthologs from the three Papaver genomes and other five angiosperm species including the monocot Oryza sativa ${ }^{88}$, Aquilegia coerule $^{89}$, Macleaya cordata ${ }^{90}$, Arabidopsis thalian $a^{91}$, and Vitis vinifera ${ }^{46}$. Then, MAFFT (v7) ${ }^{92}$ was applied to align the detected single-copy ortholog pairs and the conserved sites were extracted by using Gblocks (v0.91b) ${ }^{93}$ with default parameters. The maximum likelihood phylogenomic tree was constructed by using RAxML $(\mathrm{v} 8.2 .12)^{94}$ with 100 bootstraps. Based on the neutral theory and molecular clock ${ }^{95}$, synonymous substitution rate (Ks) measures the divergence time. The divergence times between species were estimated using the Penalized likelihood (PL) method and parameter of "setsmoothing $=1000$ " with r8s v. $1.8^{96}$, based on the constructed phylogenetic tree and the fixage times of monocot-dicot split time (152 Mya, http:// timetree.org $/)^{48}$, the constrain taxon time of Aquilegia-Papaver (127.9-139.4 Mya, http://timetree.org $/)^{48}$, and the constrain taxon time of $A$. thaliana and V. vinifera (107-135 Mya, http://timetree.org/ $/)^{48}$. We estimated the P. rhoeas and P. somniferum diverged time at around 7.7 Mya, consisting of timetree website ${ }^{48}$ (http:// timetree.org/) reports. Similarly, we estimated the divergence time of P. somniferum and P. setigerum as 4.9 Mya.

Timing of whole-genome duplications. To estimate the timing of the WGD event in P. somniferum and P. setigerum, Ks values of $P$. somniferum syntenic block genes and Ks values of $P$. setigerum syntenic block genes were calculated respectively using YN model in KaKs_Calculator (v2.0 $)^{97}$. For P. setigerum, we considered the reciprocal best matches among the syntenic gene pairs as the pairs from WGD-2 (the second WGD event) while other syntenic gene pairs were grouped as the pairs from WGD-1 (the first WGD event). The Ks value distributions were then fitted to a mixture model of Gaussian distribution using the Mclust R package ${ }^{98}$. We identified components associated with WGD peaks and calculated their mean and standard deviation of Ks values. To time the WGDs in three Papaver species, we estimated the average evolutionary rate for Papaveraceae based on the estimated divergence time of 7.7 Mya and the mean Ks value (0.12) of $P$. somniferum-P. rhoeas syntenic gene pairs. We calculated the synonymous substitutions per site per year $(r)$ for Papaveraceae equaling $8.08 \mathrm{e}-9(r=K s / 2 T)$. The $r$ value and the mean WGD Ks value were applied to time the WGDs in P. somniferum and P. setigerum based on $T=K s / 2 r$.

Ancestral genome reconstruction. The pre- and post-WGD-1 ancestors were inferred according to a workflow with three stages. In the first stage, we built the syntenic relations among $P$. setigerum, $P$. somniferum, and $P$. rhoeas. The ortholog gene pairs were detected by BlastP and MCscanX ${ }^{87}$. Next, a graph was built based on the ortholog gene pairs, and the graph components were detected as ortholog gene groups (that is putative protogenes, pPGs). We defined a PPG, consisting of four genes from $P$. setigerum, two genes from $P$. somniferum, and one gene from $P$. rhoeas, as a core pPG based on the corresponding numbers of WGD. Finally, DRIMM-Synteny ${ }^{99}$ were performed on the core $\mathrm{PPG}$ to identify the nonoverlapping (NO) synteny blocks in the three Papaver species. We filtered blocks without satisfying 4:2:1 ratio in P. setigerum, P. somniferum, and P. rhoeas. And then, pPGs except for core pPGs were fill in NO synteny blocks. In stage two, the ancestor protochromosomes were reconstructed by solving GMP (genome median

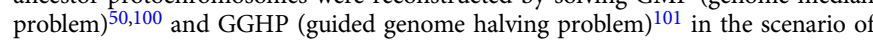
genomes with multiple WGD events based on the detected NO synteny blocks. We modeled GMP and GGHP in scenario with multiple WGDs as a block matching optimization (MO) problem based on SCoJ (single cut or join) genomic distance ${ }^{102}$, and solved this problem by an integer programming method. In the third stage, we inferred the possible order of PPGs based on the previously reconstructed ancestor protochromosomes and NO syntenic blocks. We built a directed weighted pPG graph for each syntenic block. A topological sorting method with a greedy strategy was performed on each graph to get possible gene orders. Compare to MGRA ${ }^{103}$, which is the core program in Murat et al.'s research ${ }^{47}$ and is can only apply to the block ratio satisfied 1:1:1 and not able to reconstruct ancestral genome for the evolutionary scenarios with WGD ${ }^{104}$, our pipeline adopted MO to match block copies in Papaver species by minimizing SCoJ distance to reduce the block ratio, enabling transformed problems in Papaver species (with two recent WGDs, one shared and one lineage-specific) to traditional GMP and GGHP. Our workflow can also be applied to similar evolutionary scenarios of Papaver. We are still exploring the solutions for other complex evolutionary scenarios and eventually will provide a generalized and user-friendly framework for all possible evolutionary scenarios. Our workflow is based on the accuracy of genome assembly. But now even with the cutting-edge sequencing data and widely used assembly methods, assembly errors are inevitable ${ }^{105}$. The potential misassembly may affect the reconstruction and downstream analysis. Therefore, computational evaluation and experimentally validation of genome assemblies are important to obtain more reliable results.

Gene expression analysis. The cleaned RNA reads were aligned against the assembled genome using Hisat2 (v2.2.1) ${ }^{106}$ and transcripts were discovered and quantified by Stringtie (v2.1.4) and Ballgown ${ }^{107}$, respectively, with default parameters. We measured the gene expression level by TPM (transcripts per million). To compare the TPMs between different species, we normalized the TPM by calculating $z$-score in each species. For each species, we first calculated the mean and standard deviation values of all TPMs. Then we calculated TPM $\mathrm{M}_{z}$ of each TPM as $\mathrm{TPM}_{z}=(\mathrm{TPM}-$ mean $) / \mathrm{sd}$, where the mean is the mean value of all TPMs, and sd is the standard deviation of all TPMs. Furthermore, we calculated the normalized TPM as $\mathrm{TPM}_{n}=\mathrm{TPM}_{z}+\min \mathrm{TPM}_{z}$ to make the normalized TPM nonnegative, where min_TPM ${ }_{z}$ is the minimize value of all $\mathrm{TPM}_{z}$.

Hi-C data analysis. Juicer (v1.6) $)^{108}$ and Tadtools (v0.76) ${ }^{109}$ were used to calculating Hi-C contact matrix and generating the heatmap. The chromatin loops were detected by HICCUPS ${ }^{110}$.

Reporting summary. Further information on research design is available in the Nature Research Reporting Summary linked to this article.

\section{Data availability}

Data supporting the findings of this work are available within the paper and its Supplementary Information files. A reporting summary for this Article is available as a Supplementary Information file. The Oxford Nanopore, Illumina paired-end, Hi-C, transcriptome sequencing data generated in this study have been deposited in the NCBI Sequence Read Archive (SRA) database under accession code PRJNA720042 and the National Genomics Data Center under accession code PRJCA004217. This study used previously published RNA-seq data under accession number GSE11111924. The genome assembly data are available at the Genome Warehouse in National Genomics Data Center under accession number GWHAZPI00000000, GWHAZPH00000000, and GWHAZPJ00000000 for $P$. rhoeas genome, $P$. setigerum genome and $P$. somniferum genome, respectively. The genome annotations of three Papaver genomes are available from GitHub [https://xjtu-omics.github.io/Papaver-Genomics]. The used genomes and annotations of Oryza sativa ${ }^{88}$ [http://plants.ensembl.org/Oryza_sativa/Info/Index], Arabidopsis thaliana ${ }^{91}$ [http://plants.ensembl.org/Arabidopsis_thaliana/Info/Index], and Vitis vinifera ${ }^{46}$ [http://plants.ensembl.org/Vitis_vinifera/Info/Index] are downloaded from EnsemblPlants database. The used genomes and annotations of Aquilegia coerulea $^{89}$ are downloaded from Phytozome database [https://phytozomenext.jgi.doe.gov/info/Acoerulea_v3_1]. The used genomes and annotations of Macleaya cordata $^{90}$ are downloaded from the GenBank database under accession number GCA_002174775.1. Source data are provided with this paper.

\section{Code availability}

The ancestral genome reconstruction code has been deposited to https:/github.com/ XJTU-YeLab/IAG ${ }^{111}$ and https://doi.org/10.5281/zenodo.5528515. The analysis scripts have been deposited to https://github.com/XJTU-YeLab/Papaver-Genomics/tree/main/ analysis_scripts ${ }^{112}$ and https://doi.org/10.5281/zenodo.5528517.

Received: 21 March 2021; Accepted: 29 September 2021; Published online: 15 October 2021 


\section{References}

1. Field, B. \& Osbourn, A. E. Metabolic diversification-independent assembly of operon-like gene clusters in different plants. Science 320, 543-547 (2008).

2. Winzer, T. et al. A Papaver somniferum 10-gene cluster for synthesis of the anticancer alkaloid noscapine. Science 336, 1704-1708 (2012).

3. Itkin, M. et al. Biosynthesis of antinutritional alkaloids in solanaceous crops is mediated by clustered genes. Science 341, 175-179 (2013).

4. Wilderman, P. R., Xu, M., Jin, Y., Coates, R. M. \& Peters, R. J. Identification of syn-pimara-7,15-diene synthase reveals functional clustering of terpene synthases involved in rice phytoalexin/allelochemical biosynthesis. Plant Physiol. 135, 2098-2105 (2004).

5. Jonczyk, R. et al. Elucidation of the final reactions of DIMBOA-glucoside biosynthesis in maize: characterization of $\mathrm{Bx} 6$ and Bx7. Plant Physiol. 146, 1053-1063 (2008).

6. Xiong, X. et al. The Taxus genome provides insights into paclitaxel biosynthesis. Nat. Plants https://doi.org/10.1038/s41477-021-00963-5 (2021)

7. Kautsar, S. A., Suarez Duran, H. G., Blin, K., Osbourn, A. \& Medema, M. H. plantiSMASH: automated identification, annotation and expression analysis of plant biosynthetic gene clusters. Nucleic Acids Res. 45, W55-W63 (2017).

8. Topfer, N., Fuchs, L. M. \& Aharoni, A. The PhytoClust tool for metabolic gene clusters discovery in plant genomes. Nucleic Acids Res. 45, 7049-7063 (2017).

9. Nutzmann, H. W., Scazzocchio, C. \& Osbourn, A. Metabolic gene clusters in eukaryotes. Annu Rev. Genet. 52, 159-183 (2018).

10. Takos, A. M. \& Rook, F. Why biosynthetic genes for chemical defense compounds cluster. Trends Plant Sci. 17, 383-388 (2012).

11. Hurst, L. D., Pal, C. \& Lercher, M. J. The evolutionary dynamics of eukaryotic gene order. Nat. Rev. Genet. 5, 299-310 (2004).

12. Wong, S. \& Wolfe, K. H. Birth of a metabolic gene cluster in yeast by adaptive gene relocation. Nat. Genet. 37, 777-782 (2005).

13. Slot, J. C. \& Rokas, A. Multiple GAL pathway gene clusters evolved independently and by different mechanisms in fungi. Proc. Natl Acad. Sci. USA 107, 10136-10141 (2010).

14. Rosewich, U. L. \& Kistler, H. C. Role of horizontal gene transfer in the evolution of fungi. Annu. Rev. Phytopathol. 38, 325-363 (2000).

15. Rokas, A., Wisecaver, J. H. \& Lind, A. L. The birth, evolution and death of metabolic gene clusters in fungi. Nat. Rev. Microbiol. 16, 731-744 (2018).

16. Norn, S., Kruse, P. R. \& Kruse, E. History of opium poppy and morphine. Dan. Med. Arbog. 33, 171-184 (2005).

17. Zhu, L. \& Chen, L. Progress in research on paclitaxel and tumor immunotherapy. Cell Mol. Biol. Lett. 24, 40 (2019).

18. Mao, L. et al. Genomic evidence for convergent evolution of gene clusters for momilactone biosynthesis in land plants. Proc. Natl Acad. Sci. USA 117, 12472-12480 (2020).

19. Chen, S., Krinsky, B. H. \& Long, M. New genes as drivers of phenotypic evolution. Nat. Rev. Genet. 14, 645-660 (2013).

20. Long, M., Betran, E., Thornton, K. \& Wang, W. The origin of new genes: glimpses from the young and old. Nat. Rev. Genet. 4, 865-875 (2003).

21. Kaessmann, H. Origins, evolution, and phenotypic impact of new genes. Genome Res. 20, 1313-1326 (2010).

22. Qi, X. et al. A gene cluster for secondary metabolism in oat: implications for the evolution of metabolic diversity in plants. Proc. Natl Acad. Sci. USA 101, 8233-8238 (2004).

23. Leong, B. J. \& Last, R. L. Promiscuity, impersonation and accommodation: evolution of plant specialized metabolism. Curr. Opin. Struct. Biol. 47, 105-112 (2017).

24. Guo, L. et al. The opium poppy genome and morphinan production. Science 362, 343-347 (2018).

25. Liu, Z. et al. Formation and diversification of a paradigm biosynthetic gene cluster in plants. Nat. Commun. 11, 5354 (2020).

26. Boutanaev, A. M. \& Osbourn, A. E. Multigenome analysis implicates miniature inverted-repeat transposable elements (MITEs) in metabolic diversification in eudicots. Proc. Natl Acad. Sci. USA 115, E6650-E6658 (2018).

27. Granick, S. The structural and functional relationships between heme and chlorophyll. Harvey Lect. Ser. 44, 220-245 (1948).

28. Granick, S. Speculations on the origins and evolution of photosynthesis. Ann. N. Y. Acad. Sci. 69, 292-308 (1957).

29. Horowitz, N. H. On the evolution of biochemical syntheses. Proc. Natl Acad. Sci. USA 31, 153-157 (1945).

30. Jensen, R. A. Enzyme recruitment in evolution of new function. Annu. Rev. Microbiol. 30, 409-425 (1976).

31. Ycas, M. On earlier states of the biochemical system. J. Theor. Biol. 44, 145-160 (1974).

32. Beaudoin, G. A. \& Facchini, P. J. Benzylisoquinoline alkaloid biosynthesis in opium poppy. Planta 240, 19-32 (2014).

33. Hagel, J. M. \& Facchini, P. J. Benzylisoquinoline alkaloid metabolism: a century of discovery and a brave new world. Plant Cell Physiol. 54, 647-672 (2013).
34. Menendez-Perdomo, I. M. \& Facchini, P. J. Isolation and characterization of two O-methyltransferases involved in benzylisoquinoline alkaloid biosynthesis in sacred lotus (Nelumbo nucifera). J. Biol. Chem. 295, 1598-1612 (2020).

35. Galanie, S., Thodey, K., Trenchard, I. J., Filsinger Interrante, M. \& Smolke, C. D. Complete biosynthesis of opioids in yeast. Science 349, 1095-1100 (2015).

36. Farrow, S. C., Hagel, J. M., Beaudoin, G. A., Burns, D. C. \& Facchini, P. J. Stereochemical inversion of (S)-reticuline by a cytochrome P450 fusion in opium poppy. Nat. Chem. Biol. 11, 728-732 (2015).

37. Winzer, T. et al. Morphinan biosynthesis in opium poppy requires a P450oxidoreductase fusion protein. Science 349, 309-312 (2015).

38. Singh, A., Menéndez-Perdomo, I. M. \& Facchini, P. J. Benzylisoquinoline alkaloid biosynthesis in opium poppy: an update. Phytochem. Rev. 18, 1457-1482 (2019).

39. Ziegler, J. et al. Evolution of morphine biosynthesis in opium poppy. Phytochemistry 70, 1696-1707 (2009).

40. Chen, X. et al. A pathogenesis-related 10 protein catalyzes the final step in thebaine biosynthesis. Nat. Chem. Biol. 14, 738-743 (2018).

41. Hrishi, N. J. Cytogenetical studies on Papaver somniferum L. and Papaver setigerum DC their hybrid. Genetica 31, 1-130 (1960).

42. Choe, S. et al. Species identification of Papaver by metabolite profiling. Forensic Sci. Int. 211, 51-60 (2011).

43. Asghari-Zakaria, R., Razmi, S., Madadi, R. \& Fathi, M. Karyological study of the medicinal plant Papaver rhoeas from northwest of Iran. Afr. J. Biotechnol. 10, 11173-11177 (2011)

44. Claudia, V., Mădălina, V. \& Ion, B. I. The study of mitotic chromosomes at Papaver rhoeas $1 .(2 \mathrm{n}=14)$ species. Analele tiin ifice ale Universit. Cuza din Ia (serie nou), Sec iunea I, Genetic Biologie Molecular 188-190 (2004).

45. Simao, F. A., Waterhouse, R. M., Ioannidis, P., Kriventseva, E. V. \& Zdobnov, E. M. BUSCO: assessing genome assembly and annotation completeness with single-copy orthologs. Bioinformatics 31, 3210-3212 (2015).

46. Jaillon, O. et al. The grapevine genome sequence suggests ancestral hexaploidization in major angiosperm phyla. Nature 449, 463-467 (2007)

47. Murat, F., Armero, A., Pont, C., Klopp, C. \& Salse, J. Reconstructing the genome of the most recent common ancestor of flowering plants. Nat. Genet. 49, 490-496 (2017).

48. Kumar, S., Stecher, G., Suleski, M. \& Hedges, S. B. TimeTree: a resource for timelines, timetrees, and divergence times. Mol. Biol. Evol. 34, 1812-1819 (2017).

49. Emms, D. M. \& Kelly, S. OrthoFinder: phylogenetic orthology inference for comparative genomics. Genome Biol. 20, 238 (2019).

50. Sankoff, D. \& Blanchette, M. International Computing and Combinatorics Conference 251-263 (Springer, 1997).

51. Zheng, C., Zhu, Q. \& Sankoff, D. Genome halving with an outgroup. Evol. Bioinformatics Online 2, 295-302 (2007).

52. Gaynor, M. L., Lim-Hing, S. \& Mason, C. M. Impact of genome duplication on secondary metabolite composition in non-cultivated species: a systematic meta-analysis. Ann. Bot. 126, 363-376 (2020).

53. Li, Y., Winzer, T., He, Z. \& Graham, I. A. Over 100 million years of enzyme evolution underpinning the production of morphine in the papaveraceae family of flowering plants. Plant Commun. 1, 100029 (2020).

54. Nutzmann, H. W. \& Osbourn, A. Gene clustering in plant specialized metabolism. Curr. Opin. Biotechnol. 26, 91-99 (2014).

55. Nutzmann, H. W., Huang, A. \& Osbourn, A. Plant metabolic clusters - from genetics to genomics. N. Phytol. 211, 771-789 (2016).

56. $\mathrm{Li}, \mathrm{Q}$. et al. Gene clustering and copy number variation in alkaloid metabolic pathways of opium poppy. Nat. Commun. 11, 1190 (2020).

57. Frey, M. et al. Analysis of a chemical plant defense mechanism in grasses. Science 277, 696-699 (1997)

58. Eldredge, N. Time Frames 193-224 (Princeton University Press, 2014)

59. Menendez-Perdomo, I. M., Hagel, J. M. \& Facchini, P. J. Benzylisoquinoline alkaloid analysis using high-resolution Orbitrap LC-MS(n). J. Mass Spectrom. 56, e4683 (2021).

60. Grauso, L., de Falco, B., Motti, R. \& Lanzotti, V. Corn poppy, Papaver rhoeas L.: a critical review of its botany, phytochemistry and pharmacology. Phytochem. Rev. 20, 227-248 (2021).

61. Granick, S. Evolving Genes and Proteins 67-88 (Elsevier, 1965).

62. Rison, S. C. \& Thornton, J. M. Pathway evolution, structurally speaking. Curr. Opin. Struct. Biol. 12, 374-382 (2002).

63. Copley, S. D. Evolution of a metabolic pathway for degradation of a toxic xenobiotic: the patchwork approach. Trends Biochem. Sci. 25, 261-265 (2000).

64. Liu, H. et al. Patchwork assembly of nag-like nitroarene dioxygenase genes and the 3-chlorocatechol degradation cluster for evolution of the 2-chloronitrobenzene catabolism pathway in Pseudomonas stutzeri ZWLR2-1. Appl. Environ. Microbiol. 77, 4547-4552 (2011).

65. Nett, R. S., Lau, W. \& Sattely, E. S. Discovery and engineering of colchicine alkaloid biosynthesis. Nature 584, 148-153 (2020).

66. Zhou, J., Bruns, M. A. \& Tiedje, J. M. DNA recovery from soils of diverse composition. Appl. Environ. Microbiol. 62, 316-322 (1996). 
67. Louwers, M. et al. Tissue- and expression level-specific chromatin looping at maize b1 epialleles. Plant Cell 21, 832-842 (2009).

68. Wick, R. R. \& Holt, K. E. Benchmarking of long-read assemblers for prokaryote whole genome sequencing. F1000Res 8, 2138 (2019).

69. Hu, J., Fan, J., Sun, Z. \& Liu, S. NextPolish: a fast and efficient genome polishing tool for long-read assembly. Bioinformatics 36, 2253-2255 (2020).

70. Guan, D. et al. Identifying and removing haplotypic duplication in primary genome assemblies. Bioinformatics 36, 2896-2898 (2020).

71. Dudchenko, O. et al. De novo assembly of the Aedes aegypti genome using HiC yields chromosome-length scaffolds. Science 356, 92-95 (2017).

72. McKenna, A. et al. The Genome Analysis Toolkit: a MapReduce framework for analyzing next-generation DNA sequencing data. Genome Res. 20, 1297-1303 (2010).

73. Bao, W., Kojima, K. K. \& Kohany, O. Repbase update, a database of repetitive elements in eukaryotic genomes. Mob. DNA 6, 11 (2015).

74. Xu, Z. \& Wang, H. LTR_FINDER: an efficient tool for the prediction of fulllength LTR retrotransposons. Nucleic Acids Res. 35, W265-W268 (2007).

75. Ellinghaus, D., Kurtz, S. \& Willhoeft, U. LTRharvest, an efficient and flexible software for de novo detection of LTR retrotransposons. BMC Bioinformatics 9, 18 (2008).

76. Ou, S. \& Jiang, N. LTR_retriever: a highly accurate and sensitive program for identification of long terminal repeat retrotransposons. Plant Physiol. 176, 1410-1422 (2018).

77. Campbell, M. S., Holt, C., Moore, B. \& Yandell, M. Genome annotation and curation using MAKER and MAKER-P. Curr. Protoc. Bioinformatics 48, 411 11-39 (2014)

78. Lamesch, P. et al. The Arabidopsis Information Resource (TAIR): improved gene annotation and new tools. Nucleic Acids Res. 40, D1202-D1210 (2012).

79. Dohm, J. C. et al. The genome of the recently domesticated crop plant sugar beet (Beta vulgaris). Nature 505, 546-549 (2014).

80. Grabherr, M. G. et al. Full-length transcriptome assembly from RNA-Seq data without a reference genome. Nat. Biotechnol. 29, 644-652 (2011)

81. Keller, O., Kollmar, M., Stanke, M. \& Waack, S. A novel hybrid gene prediction method employing protein multiple sequence alignments. Bioinformatics 27, 757-763 (2011).

82. Korf, I. Gene finding in novel genomes. BMC Bioinformatics 5, 59 (2004).

83. Lomsadze, A., Ter-Hovhannisyan, V., Chernoff, Y. O. \& Borodovsky, M. Gene identification in novel eukaryotic genomes by self-training algorithm. Nucleic Acids Res. 33, 6494-6506 (2005).

84. Jones, P. et al. InterProScan 5: genome-scale protein function classification. Bioinformatics 30, 1236-1240 (2014).

85. Nawrocki, E. P. Annotating functional RNAs in genomes using Infernal. Methods Mol. Biol. 1097, 163-197 (2014).

86. Nawrocki, E. P. et al. Rfam 12.0: updates to the RNA families database. Nucleic Acids Res. 43, D130-D137 (2015).

87. Wang, Y. et al. MCScanX: a toolkit for detection and evolutionary analysis of gene synteny and collinearity. Nucleic Acids Res. 40, e49 (2012).

88. International Rice Genome Sequencing Project. The map-based sequence of the rice genome. Nature 436, 793-800 (2005).

89. Filiault, D. L. et al. The Aquilegia genome provides insight into adaptive radiation and reveals an extraordinarily polymorphic chromosome with a unique history. Elife 7, https://doi.org/10.7554/eLife.36426 (2018).

90. Liu, X. et al. The Genome of medicinal plant Macleaya cordata provides new insights into benzylisoquinoline alkaloids metabolism. Mol. Plant 10, 975-989 (2017).

91. Cheng, C. Y. et al. Araport11: a complete reannotation of the Arabidopsis thaliana reference genome. Plant J. 89, 789-804 (2017).

92. Katoh, K. \& Standley, D. M. MAFFT multiple sequence alignment software version 7: improvements in performance and usability. Mol. Biol. Evol. 30, 772-780 (2013).

93. Castresana, J. Selection of conserved blocks from multiple alignments for their use in phylogenetic analysis. Mol. Biol. Evol. 17, 540-552 (2000).

94. Stamatakis, A. RAxML-VI-HPC: maximum likelihood-based phylogenetic analyses with thousands of taxa and mixed models. Bioinformatics 22, 2688-2690 (2006).

95. Kimura, M. Molecular evolutionary clock and the neutral theory. J. Mol. Evol. 26, 24-33 (1987).

96. Sanderson, M. J. r8s: inferring absolute rates of molecular evolution and divergence times in the absence of a molecular clock. Bioinformatics 19, 301-302 (2003).

97. Wang, D., Zhang, Y., Zhang, Z., Zhu, J. \& Yu, J. KaKs_Calculator 2.0: a toolkit incorporating gamma-series methods and sliding window strategies. Genomics Proteom. Bioinformatics 8, 77-80 (2010).

98. Scrucca, L., Fop, M., Murphy, T. B. \& Raftery, A. E. mclust 5: clustering, classification and density estimation using Gaussian finite mixture models. $R$. J. 8, 289-317 (2016).

99. Pham, S. K. \& Pevzner, P. A. DRIMM-Synteny: decomposing genomes into evolutionary conserved segments. Bioinformatics 26, 2509-2516 (2010).
100. Tannier, E., Zheng, C. \& Sankoff, D. Multichromosomal median and halving problems under different genomic distances. BMC Bioinformatics 10, 120 (2009)

101. Zheng, C., Zhu, Q., Adam, Z. \& Sankoff, D. Guided genome halving: hardness, heuristics and the history of the Hemiascomycetes. Bioinformatics 24, i96-i104 (2008).

102. Feijao, P. \& Meidanis, J. SCJ: a breakpoint-like distance that simplifies severa rearrangement problems. IEEE/ACM Trans. Comput. Biol. Bioinformatics $\mathbf{8}$, 1318-1329 (2011)

103. Alekseyev, M. A. \& Pevzner, P. A. Breakpoint graphs and ancestral genome reconstructions. Genome Res. 19, 943-957 (2009).

104. Anselmetti, Y., Luhmann, N., Berard, S., Tannier, E. \& Chauve, C. Comparative methods for reconstructing ancient genome organization. Methods Mol. Biol. 1704, 343-362 (2018).

105. Rai, A. et al. Chromosome-level genome assembly of Ophiorrhiza pumila reveals the evolution of camptothecin biosynthesis. Nat. Commun. 12, 405 (2021).

106. Kim, D., Paggi, J. M., Park, C., Bennett, C. \& Salzberg, S. L. Graph-based genome alignment and genotyping with HISAT2 and HISAT-genotype. Nat. Biotechnol. 37, 907-915 (2019).

107. Pertea, M., Kim, D., Pertea, G. M., Leek, J. T. \& Salzberg, S. L. Transcript-level expression analysis of RNA-seq experiments with HISAT, StringTie and Ballgown. Nat. Protoc. 11, 1650-1667 (2016).

108. Durand, N. C. et al. Juicer provides a one-click system for analyzing loopresolution Hi-C experiments. Cell Syst. 3, 95-98 (2016).

109. Kruse, K., Hug, C. B., Hernandez-Rodriguez, B. \& Vaquerizas, J. M. TADtool visual parameter identification for TAD-calling algorithms. Bioinformatics $\mathbf{3 2}$ 3190-3192 (2016).

110. Rao, S. S. et al. A 3D map of the human genome at kilobase resolution reveals principles of chromatin looping. Cell 159, 1665-1680 (2014).

111. Yang, X. et al. Three chromosome-scale Papaver genomes reveal punctuated patchwork evolution of the morphinan and noscapine biosynthesis pathway. Zenodo https://doi.org/10.5281/zenodo.5528515 (2021).

112. Yang, X. et al. Three chromosome-scale Papaver genomes reveal punctuated patchwork evolution of the morphinan and noscapine biosynthesis pathway. Zenodo https://doi.org/10.5281/zenodo.5528517 (2021)

\section{Acknowledgements}

We thank Ian A. Graham, Yi Li, Wen Wang, and Zemin Ning for helpful discussions on genome analysis, and Jing Hai, Langchong He, Shengli Han, and Xumei Wang for administrative and technical support. X.Y., K.Y., and L.G. are supported by the National Science Foundation of China $(62172325,32125009,32070663$, 31970317), the Key Construction Program of the National '985' Project, and the Fundamental Research Funds for the Central Universities.

\section{Author contributions}

K.Y. designed and supervised research. L.G. and Y.J. collected materials for sequencing. X.Y., S.G, P.J., and T.X. performed the genome assembly, assembly evaluation, genome annotation, and synteny analysis. K.Y., X.Y., S.G., and L.G. interpreted the WGD events. X.Y. and T.X. performed the gene expression analysis. X.Y. and B.W. performed phylogenomic analysis. S.G., X.Y., J.L., and J.S. performed ancestral genome reconstruction. Y.J. and J.Z. performed the karyotyping of three species. X.Y. and Y.C. performed the Hi-C data analysis. K.Y., X.Y., L.G., and S.G. interpreted the evolution of the BIA gene cluster. X.Y., K.Y., S.G., and Y.C. prepared figures and tables. K.Y., X.Y., L.G., and S.G. wrote the paper. All authors read and proved the final version of this manuscript.

\section{Competing interests}

The authors declare no competing interests.

\section{Additional information}

Supplementary information The online version contains supplementary material available at https://doi.org/10.1038/s41467-021-26330-8.

Correspondence and requests for materials should be addressed to Kai Ye.

Peer review information Nature Communications thanks Thu-Thuy Dang, Elisabeth Kaltenegger, Amit Rai and the other, anonymous, reviewer(s) for their contribution to the peer review of this work. Peer reviewer reports are available.

Reprints and permission information is available at http://www.nature.com/reprints

Publisher's note Springer Nature remains neutral with regard to jurisdictional claims in published maps and institutional affiliations. 
(c) (i) Open Access This article is licensed under a Creative Commons Attribution 4.0 International License, which permits use, sharing, adaptation, distribution and reproduction in any medium or format, as long as you give appropriate credit to the original author(s) and the source, provide a link to the Creative Commons license, and indicate if changes were made. The images or other third party material in this article are included in the article's Creative Commons license, unless indicated otherwise in a credit line to the material. If material is not included in the article's Creative Commons license and your intended use is not permitted by statutory regulation or exceeds the permitted use, you will need to obtain permission directly from the copyright holder. To view a copy of this license, visit http://creativecommons.org/ licenses/by/4.0/.

(C) The Author(s) 2021 